\title{
MODĖLES ET ANALYSE DE RÉGIMES D’INVENTION DANS LE DROIT DU BREVET AMÉRICAIN (1790-2007)
}

\section{Chipten Valibhay, Pascal Le Masson, Benoît Weil}

\author{
ESKA | Entreprises et histoire »
}

2020/1 nº 98 | pages 42 à 77

ISSN 1161-2770

ISBN 9782747230445

\section{Article disponible en ligne à l'adresse :}

https://www.cairn.info/revue-entreprises-et-histoire-2020-1-page-42.htm

Distribution électronique Cairn.info pour ESKA.

(C) ESKA. Tous droits réservés pour tous pays.

La reproduction ou représentation de cet article, notamment par photocopie, n'est autorisée que dans les limites des conditions générales d'utilisation du site ou, le cas échéant, des conditions générales de la licence souscrite par votre établissement. Toute autre reproduction ou représentation, en tout ou partie, sous quelque forme et de quelque manière que ce soit, est interdite sauf accord préalable et écrit de l'éditeur, en dehors des cas prévus par la législation en vigueur en France. Il est précisé que son stockage dans une base de données est également interdit. 


\section{MODĖLES ET ANALYSE DE RÉGIMES D'INVENTION DANS LE DROIT DU BREVET AMÉRICAIN (1790-2007)}

par Chipten VALIBHAY

Doctorant CIFRE en sciences de gestion, Institut National de la Propriété Industrielle

- Centre de Gestion Scientifique, MINES ParisTech, PSL Université

\section{Pascal LE MASSON}

Professeur de sciences de gestion, MINES ParisTech, PSL Université, Centre de Gestion Scientifique

\section{et Benoît WEIL}

Professeur de sciences de gestion, MINES ParisTech, PSL Université, Centre de Gestion Scientifique

Que nous disent les brevets sur l'histoire des inventions? C'est par l'intermédiaire du droit que cet article aborde la question, en étudiant les décisions de justice aux États-Unis entre la fin du XVIIIe et le début du $X X l^{e}$ siècle. En décidant comment le connu et l'inconnu pouvaient s'articuler à différentes périodes, les juges américains ont écrit une histoire des inventions distincte d'une simple histoire du progrès technique.

\section{INTRODUCTION}

Dès les années 1930, Schumpeter a opéré une distinction, maintes fois reprise depuis, entre invention et innovation. L'économiste trace une frontière entre les deux phénomènes en expliquant que « l'invention en elle-même ne peut induire l'innovation $»^{1}$, et que les processus qui les produisent sont de nature différente. Si les processus d'innovation sont devenus des objets d'étude pour ses successeurs, l'invention n'a pas connu le même sort. Le brevet, forme juridique de l'invention, occupe pourtant une place primordiale dans les stratégies d'innovation, et il s'est imposé comme un observable majeur des évolutions techniques. Cet écart de traitement entre brevet et invention interroge, et semble révéler un point aveugle : la notion d'invention elle-même est souvent éludée. En quoi le brevet incorporet-il une trace de ce qui est « inventif »? Quelle représentation de l'invention retient le brevet ? Et, plus généralement, qu'appelle-t-on « invention technique»? Les travaux portant

${ }^{1}$ J. Schumpeter, Business Cycles: A Theoretical, Historical, and Statistical Analysis of the Capitalist Process, New York, McGraw-Hill, 1939, p. 80. 
sur la gestion ou l'économie de l'innovation abordent peu ces questions, l'invention étant analysée comme une ressource-connaissance, savoir-faire ou actif technologique, qu'il s'agit de valoriser plutôt que de caractériser. En ce sens, la nature " inventive » de cet objet disparaît et l'activité d'invention se trouve réduite à une création de nouvelles connaissances. Une meilleure caractérisation de ce phénomène " inventif » permettrait de mieux saisir l'acte d'invention mais aussi de mieux comprendre certaines des propriétés de cette infrastructure inventive - les brevets en particulier - à partir de laquelle se construit et s'étudie l'innovation technologique.

Discuter de ce qu'est l'invention au sens du brevet offre un avantage certain : le droit de la propriété intellectuelle et le système des brevets fournissent un corpus de références traitant précisément des inventions, constitué de lois, de directives et d'une jurisprudence abondante. Plus important encore, lors de l'examen des brevets au sein des systèmes nationaux et internationaux, les inventions sont évaluées de manière régulière et systématique par les examinateurs de brevets lors du dépôt ou par les juges lors des contentieux. Comprendre et modéliser les logiques d'évaluation des acteurs de l'invention pourrait donc constituer une ressource unique pour mieux comprendre l'invention et former les inventeurs. En somme, comprendre ce qu'est une « bonne invention » donnerait les moyens de bien inventer. Les critères d'évaluation existent - les critères de brevetabilité - et l'évaluation des inventions au travers de ces critères est une activité organisée, répétée et extrêmement intense (plus de 8000 examinateurs de brevets au United States Patent and Trademark Office [USPTO] ${ }^{2}$ !). On a donc là l'occasion de saisir en détail ce que signifie « évaluer une invention » et d'en déduire ce qu'est une « invention validée ». Ajoutons que cette approche permet étonnamment de penser l'invention indépendamment de son créateur: l'invention est ici définie et évaluée par un récepteur-l'examinateur-qui juge de l'inventivité, sans connaître le raisonnement réel du concepteur. Dans cette perspective, l'invention ne peut être décrite par une théorie de la créativité (associée au créateur) et oblige à développer d'autres formes originales de caractérisation du raisonnement inventif et à dépasser un étrange paradoxe : comment caractériser une exploration dans l'inconnu (l'acte inventif) en n'ayant accès qu'au connu (savoir préexistant à l'invention et le savoir qui résulte de l'invention)?

L'étude des pratiques d'évaluation n'est donc pas si simple à mettre en œuvre. On pourrait par ailleurs penser que la notion d'invention est d'autant plus stable qu'elle est fondée en droit. Est-ce certain ? On est ainsi surpris de voir certains juristes rappeler que le droit du brevet est l'un des domaines du droit qui évoluent le plus régulièrement ${ }^{3}-$ c'est le $^{\prime}$ cas notamment du critère d'activité inventive, très fluctuant alors qu'il est d'une importance majeure dans le système des brevets ${ }^{4}$. Ce critère au nom caractéristique, évaluant précisément la qualité « inventive » d'un brevet, est l'objet d'étude central de cet article. Au travers de ce critère, l'histoire du droit du brevet révèle des évolutions importantes de la notion d'invention. On distingue ainsi plusieurs définitions historiques de l'invention, qui se traduisent par des critères autant que par des pratiques d'évaluation de l'invention de la part des experts des brevets et de la jurisprudence. Dans ces pratiques, l'évaluation du critère d'activité inventive repose sur une fiction juridique centrale, "l'homme du métier ", à partir de laquelle on détermine si une invention respecte ou non le critère d'activité inventive. L'étude de ces pratiques revient donc potentiellement à mettre en évidence des modèles très différents de l'homme du métier, et donc de représentation du connu. En particulier, l'un des enjeux relatifs à l'invention repose sur la capacité du

\footnotetext{
2 «Performance and Accountability Report », USPTO, 2018, p. 12.

${ }^{3}$ J. F. Duffy, « Inventing Invention: a Case Study of Legal Innovation », Texas Law Review, vol. 86, n 1, 2007, p. 1-72.

${ }^{4}$ R. P. Merges, « Uncertainty and the Standard of Patentability », High Technology Law Journal, vol. 7, n 1, 1992, p. $1-70$.
} 
concepteur à s'écarter de l'état de l'art, créant ainsi un objet nouveau. Les modèles ont donc vocation à expliciter les critères d'évaluation de cet écart, traçant la frontière entre l'invention et la non-invention. Ce sont ainsi plusieurs régimes d'invention correspondant à différentes typologies d'homme du métier et d'état de l'art correspondant qu'il serait possible d'identifier au travers de l'histoire de l'évaluation du brevet. Ce faisant on caractérise alors à la fois : 1) une façon de modéliser, à un moment donné voire dans un secteur ou une industrie donné(e), l'état de l'art et la figure d'homme du métier correspondante, et 2) une variété de critères qui déterminent la façon de s'écarter du connu et donc un régime d'invention spécifique.

À partir de l'étude généalogique du critère d'activité inventive, nous souhaiterions donc montrer comment les pratiques d'examen du brevet témoignent en creux des représentations diverses et variables dans le temps de ce qu'est l'invention, et donc mettre en évidence des formes variées d'exploration de l'inconnu. Nous discuterons la manière dont le droit et les juristes se sont emparés de la question de l'activité inventive puis nous proposerons un cadre d'analyse issu d'une théorie de la conception, la théorie $\mathrm{C} / \mathrm{K}$, permettant de définir les propriétés générales de l'invention. En menant notre étude des décisions de jurisprudence en fonction de ces propriétés, nous mettons en évidence l'existence de trois régimes d'invention spécifiques, correspondant simultanément à des structures de l'état de l'art spécifiques et à des systèmes de règles permettant d'évaluer s'il y a invention. Au-delà d'une meilleure définition du raisonnement
« inventif », c'est une histoire de la notion d'invention elle-même qui se dessine, rendant compte de l'importance, parfois négligée, des acteurs du système des brevets - examinateurs et juges de brevets - dans l'organisation et l'histoire des écosystèmes d'inventeurs.

\section{LA DÉFINITION CONTROVERSÉE DE L'INVENTION DANS LE DROIT DU BREVET}

Le phénomène « inventif » a déjà été analysé par d'importants travaux qui ont permis d'en décrire des traits significatifs, et ce à travers de nombreuses approches disciplinaires : sociologie, psychologie, économie, sciences de gestion, philosophie et histoire. Il ne s'agit pas ici d'en offrir une synthèse mais de constater qu'à notre connaissance, ces approches n'ont pas ou peu pris en compte le rôle du droit des brevets dans la définition de l'invention. Les études portant sur l'histoire des systèmes de brevets ont plus récemment mis l'accent sur l'importance d'un écosystème d'acteurs - société d'experts, savants, agents de brevets, juges et législateurs - qui a accompagné, encadré voire modulé les activités des inventeurs ${ }^{5}$. Ces nouveaux éléments poussent à considérer et modéliser les formes de l'invention prescrites par le droit du brevet ${ }^{6}$.

Depuis la création des systèmes nationaux de brevets à la fin du XVIII ${ }^{\mathrm{e}}$ siècle, se pose aux législateurs la question de la frontière

\footnotetext{
${ }^{5}$ La littérature traitant de l'histoire du droit des brevets est abondante. Cependant le rapport entre une théorie de l'invention et ce droit a été peu abordé. Les auteurs analysent davantage le droit du brevet comme un droit de propriété et un système de récompense ou de légitimation des inventeurs et mettent en avant les controverses économiques (débat relatif au droit de monopole) ou industrielles (débat relatif à la paternité des inventions par exemple). Voir : pour le cas français, G. Galvez-Behar, La République des inventeurs : inventer et posséder l'invention en France (1791-1922), Rennes, Presses Universitaires de Rennes, 2008 ou L. Hilaire-Pérez, L'invention technique au siècle des Lumières, Paris, Albin Michel, 2000 ; pour le cas anglais, C. Macleod, Inventing the Industrial Revolution: The English Patent System, 1660-1800, Cambridge, Cambridge University Press, 2002, en particulier le chapitre 11, p. 201-222: 1'auteur y décrit certaines doctrines philosophiques dominantes concernant l'invention et la technique en Angleterre; et plus généralement, sur les conditions intellectuelles de l'émergence des systèmes de brevets : M. Buydens, La propriété intellectuelle : évolution historique et philosophie, Bruxelles, Éditions Bruylant, 2012.

${ }^{6}$ Pour une approche similaire, voir C. C. Cooper, « Social construction of Invention through Patent Management: Thomas Blanchard's Woodworking Machinery », Technology and Culture, vol. 32, n 4, 1991, p. 960-998.
} 
du brevetable : la nature et la qualité des inventions est un sujet de discussion, comme en témoigne le Patent Act de 1790 aux ÉtatsUnis qui liste déjà les principaux objets pouvant donner droit à une lettre de patente en mettant en avant deux qualités : être utile et ne pas être connu ou utilisé avant la date de la demande, donc nouveau ${ }^{7}$. Aujourd'hui, ces deux critères de brevetabilité ont été conservés mais s'est ajouté un troisième critère : l'activité inventive ou «nonobviousness criteria » aux États-Unis, aujourd'hui formulé ainsi dans le droit américain :

«A patent for a claimed invention may not be obtained, notwithstanding that the claimed invention is not identically disclosed as set forth in section 102, if the differences between the claimed invention and the prior art are such that the claimed invention as a whole would have been obvious before the effective filing date of the claimed invention to a person having ordinary skill in the art to which the claimed invention pertains. Patentability shall not be negated by the manner in which the invention was made $»^{8}$.

Dans le contexte des controverses autour de la valeur des brevets et donc de leur évaluation 9 , le critère d'activité inventive est devenu un sujet d'étude important dans la littérature juridique ${ }^{10}$. Il est considéré par les juristes comme un seuil mesurant l'inventivité des brevets. Le critère apparaît dans la jurisprudence américaine en 1851 lors du procès Hotchkiss v. Greenwood ${ }^{11}$ qui en pose les premières pierres. La notion d' « inventivité » dans le droit de la propriété intellectuelle, qui paraît presque intuitive aujourd'hui, s'est construite au travers d'une question essentielle : comment distinguer l'invention de la modification mineure ou de la simple amélioration d'une solution existante? Des travaux retracent ainsi l'histoire du critère d'activité inventive et montrent qu'une figure particulière, « l'homme du métier », occupe une place majeure dans les discours ("person having ordinary skill in the art» dans les termes américains) ${ }^{12}$. Si cette figure se constitue comme artefact juridique pour l'évaluation de l'activité inventive aux ÉtatsUnis, son développement est influencé par les pratiques d'autres pays. Aux origines du système américain des brevets, les décisions de jurisprudence citent d'ailleurs régulièrement les jugements anglais comme référence ${ }^{13}$.

L'utilisation de la fiction juridique d' « homme du métier » par les examinateurs de brevets ou les juges nourrit en fait un débat animé $^{14}$. La discussion s'organise notamment autour de sa définition - qui est-il ? que sait-il ? quel est son niveau de compétences ? D'autres

\footnotetext{
${ }^{7}$ Patent Act, « An Act to Promote the Progress of Useful Arts », Section 1, 10 avril 1790 : « he, she or they, hath or have invented or discovered any useful art, manufacture, engine, machine, or device, or any improvement therein not before known or used, and praying that a patent may be granted therefor, it shall and may be lawful to and for the said Secretary of State, the Secretary for the department of war, and the Attorney General, or any two of them, if they shall deem the invention or discovery sufficiently useful and important, to cause letters patent to be issued ».

${ }^{8}$ Title 35 of the United States Codes, Section 103.

${ }^{9}$ A. B. Jaffe et J. Lerner, « Innovation and its Discontents », in Id. (eds.), Innovation Policy and the Economy, vol. 6, Cambridge (Mass.), MIT Press, 2006, p. 27-66.

${ }^{10}$ G. Mandel, «The Non-Obvious Problem: How the Indeterminate Nonobviousness Standard Produces Excessive Patent Grants », UC Davis Law Review, vol. 42, 2008, p. 57-128 ; H. V. J. Moir, «Empirical Evidence on the Inventive Step », European Intellectual Property Review, vol. 35, n 5, avril 2013, p. 246-252.

${ }^{11}$ Hotchkiss v. Greenwood, 52 U.S. 248, 13 L. Ed. 683, 11 How. 248, 1850 U.S. LEXIS 1507.

${ }^{12}$ Voir J. F. Duffy, « Inventing Invention: a Case Study of Legal Innovation », art. cit.

${ }^{13}$ W. Carpmael, Law Reports of Patent Cases, Volume I, Londres, A. Mcintosh, 1843 ; C. L. Woodbury et G. Minot, Reports of Cases Argued and Determined in the Circuit Court of the United States for the First Circuit, (1812-1875), Boston, C. C. Little \& J. Brown, 1880.

${ }^{14}$ D. L. Burk et M. A. Lemley, « Is Patent Law Technology-Specific? », Berkeley Technology Law Journal, vol. 17, $\mathrm{n}^{\circ}$ 4, 2002, p. 1155-1206; R. S. Eisenberg, « Obvious to Whom? Evaluating Inventions from the Perspective of PHOSITA », Berkeley Technology Law Journal, vol. 19, n 3, 2004, p. 885-906; C. C. Kennedy, " Rethinking Obviousness », Wisconsin Law Review, vol. 2015, n 4, 2015, p. 665-726.
} 
études traitent de la capacité des acteurs du système des brevets à l'employer comme référentiel objectif d'évaluation ${ }^{15}$, du niveau d'activité inventive qu'il est censé représenter ${ }^{16}$ ou de la variabilité de son application selon les secteurs industriels ${ }^{17}$. Alors que les critères de nouveauté et d'application industrielle apparaissent comme des questions factuelles, le critère d'activité inventive souffre d'une ambiguïté : l'inventivité d'une solution est-elle un fait? Si oui, qui peut établir ce fait : le juge, l'expert technique ou un autre tiers ? Si, au contraire, c'est une construction légale, quelle invention doit être favorisée ? Comment choisir en droit le « niveau d'inventivité » optimal? Or le fait est ici bien spécifique : il s'agit d'expliciter un raisonnement de conception, ce qui suppose des outils pour le décrire. Quant au droit, il présuppose qu'il serait possible d'établir une doctrine de l'invention relativement indépendante de l'objet auquel il s'applique ; or l'étude de Dan Burk et Mark Lemley ${ }^{16}$ démontre clairement que les doctrines varient, par exemple entre le domaine informatique et celui de la biotechnologie. La figure de l'homme du métier introduit donc une manière particulière d'évaluer le brevet : elle implique un raisonnement de conception hypothétique et simulé qui serait mené par un homme " sans capacité inventive " mais connaissant l'ensemble de l'état de l'art pertinent. Le raisonnement de l'homme du métier et ses connaissances révèlent alors en creux ce que le système des brevets considère comme inventif. Ce que l'homme du métier ne peut penser est inventif. Or cette pensée n'est pas stable : encore récemment, en 2007, lors de la décision KSR v. Teleflex, la Cour suprême des États-Unis déclarait : «A person of ordinary skill in the art is also a person of ordinary creativity, not an automaton ${ }^{18}$.

Des travaux ont récemment cherché à établir des ponts entre le droit du brevet et la littérature sur la créativité afin de mieux cerner cette figure ${ }^{19}$. Cependant, si cette approche éclaire certaines intuitions juridiques, elle nourrit peut-être un malentendu : elle renforce la personnification de l'homme du métier, et réduit l'attention portée au modèle de raisonnement qu'il incorpore. Comprendre l'évaluation du brevet consiste plutôt à observer les raisonnements menés lors de l'examen, plutôt que les raisonnements menés par les concepteurs (qu'ils soient « créatifs » ou non). Une autre approche a consisté à mieux saisir les différentes acceptions de l'homme du métier par les juges ${ }^{20}$ : cette méthode peut être trompeuse car elle incite à identifier des métiers « types » (mécanicien, designer, chercheur), sans se demander en quoi ces métiers (ou plutôt certaines activités de ces métiers) renvoient à des modèles de raisonnement variés. Cependant cette analyse révèle l'existence de ruptures jurisprudentielles qui impliquent de grandes modifications dans l'interprétation de la loi. Ces ruptures doivent être articulées avec les modifications institutionnelles qui touchent le système des brevets.

Aux États-Unis, en 1790, lors de l'établissement du système des brevets, l'examen préalable est effectué par le

\footnotetext{
${ }^{15}$ G. Mandel, « Patently Non-Obvious: Empirical Demonstration that the Hindsight Bias Renders Patent Decisions Irrational », Ohio State Law Journal, vol. 67, 2006, p.1391-1463 ; B. H. Graf, « Prognosis Indeterminable: How Patent Non-Obviousness Outcomes Depend Too Much on Decision-Maker », Cardozo Public Law Policy \& Ethics Journal, vol. 9, n 3, 2011, p. 567-606.

${ }^{16}$ M. J. Meurer et K. J. Strandburg, « Patent Carrots and Sticks: A Model of Nonobviousness », Lewis \& Clark Law Review, vol. 12, $\mathrm{n}^{\circ} 2,2008$, p. 547-578.

${ }^{17}$ D. L. Burk et M. A. Lemley, « Is Patent Law Technology-Specific? », art. cit.

${ }^{18}$ KSR Intern. Co. v. Teleflex Inc., 550 U.S. 398, 127 S. Ct. 1727, 167 L. Ed. 2d 705, 2007 U.S. LEXIS 4745.

${ }^{19}$ R. K. Sawyer, «Creativity, Innovation, and Obviousness », Lewis \& Clark Law Review, vol. 12, n 2, 2008, p. 461 487 ; A. L. Landers, « Ordinary Creativity in Patent Law: The Artist Within the Scientist », Missouri Law Review, vol. $75, \mathrm{n}^{\circ} 1,2010$, p. 1-77.

${ }^{20}$ J. J. Darrow, « The Neglected Dimension of Patent Law’s PHOSITA Standard », Harvard Journal of Law \& Technology, vol. 23, n 1, 2009, p. 227-258.
} 
secrétaire d'État (qui n'est alors autre que Thomas Jefferson, lui-même inventeur), le secrétaire à la Défense (Henry Knox) et le procureur général (Edmund Randolph) - ce qui suppose des évaluations ponctuelles, au cas par cas. En 1793, l'examen préalable est aboli (les brevets sont donc délivrés sur la bonne foi des inventeurs quant à la nouveauté de leurs inventions), avant d'être rétabli en 1836 avec la création d'un office des brevets et le recrutement d'un examinateur professionnel de brevets. Ces changements ont des impacts majeurs : en 1836, Charles Keller, le premier examinateur professionnel de brevets, rejette $75 \%$ des demandes qu'il reçoit ${ }^{21}$. L'introduction du critère de nonobviousness dans le Patent Act de 1952 confirme finalement l'usage de l'évaluation de l'activité inventive et amène donc les examinateurs à systématiser leur approche : ce sera l'objet de la décision Graham v. John Deere (1966) ${ }^{22}$ où la Cour suprême établit pour la première fois une série d'étapes dans l'évaluation. Plus généralement, les transformations du contexte industriel et l'organisation des débats relatifs au système des brevets influencent les règles et l'organisation à la fois de la délivrance des brevets et des procès. Cependant les analyses historiques mettent plutôt en scène des cas historiques célèbres (Watt, Bell, Edison, etc.) pour illustrer les stratégies de protection ou de légitimation de l'invention ${ }^{23}$. Paradoxalement, ces analyses nous renseignent sur les transformations de l'usage du système des brevets par les acteurs économiques mais elles témoignent peu des transformations légales ou organisationnelles relatives à l'évaluation des brevets que l'on souhaite étudier ici.

Nous souhaitons dans cet article proposer une modélisation, par période historique, des critères d'évaluation de l'activité inventive afin de mieux caractériser les raisonnements adoptés lors de l'évaluation. Nous observerons les types d'inventions admises ou non par le système des brevets, les connaissances mobilisées pour les analyser (renvoyant au contenu et à la structure de l'état de l'art et des connaissances de l'homme du métier), et les critères qui ont permis le jugement effectif. Cette modélisation systématique éclaire le rôle du système d'évaluation des brevets dans la dynamique des inventions brevetées. C'est aussi l'occasion de mieux saisir cette figure d'homme du métier en précisant ses évolutions et son utilisation dans les méthodes d'évaluation. Nous voudrions donc répondre à deux questions de recherche dans cet article :

1) À quels modèles de l'homme du métier - raisonnements et connaissances - renvoient les évolutions historiques du critère d'activité inventive depuis 1790 ?

2) Quels modèles de l'invention se dégagent alors de cette modélisation? Qu'a-t-on appelé « invention » dans le droit du brevet?

\section{MÉTHODOLOGIE : ANALYSE DE DÉCISIONS DE JURISPRUDENCE À PARTIR D'UNE THÉORIE DE LA CONCEPTION}

L'étendue temporelle de l'étude proposée, près de 220 ans d'évolution du système des brevets, nous oblige à focaliser notre étude sur certains cas «types » à partir desquels nous généraliserons nos conclusions. Le caractère exploratoire de cette étude ne peut donc prétendre à l'exhaustivité, mais permet de mettre en lumière des modèles de l'invention qui demanderont à être examinés plus avant.

\footnotetext{
${ }^{21}$ K. W. Swanson, «The Emergence of the Professional Patent Practitioner », Technology and Culture, vol. 50, $\mathrm{n}^{\circ} 3$, 2009, p. 519-548.

${ }^{22}$ Graham v. John Deere Co. of Kansas City, 383 U.S. 1, 86 S. Ct. 684, 15 L. Ed. 2d 545, 1966 U.S. LEXIS 2908.

${ }^{23}$ Par exemple : S. Arapostathis et G. Gooday, Patently Contestable: Electrical Technologies and Inventor Identities on Trial in Britain, Cambridge (Mass.), MIT Press, 2013 ; C. Beauchamp, Invented by Law: Alexander Graham Bell and the Patent that Changed America, Cambridge (Mass.), Harvard University Press, 2015.
} 
Pour rendre visibles les modèles utilisés dans le cadre du droit du brevet, nous avons choisi une analyse généalogique en nous appuyant sur les sources historiques qui témoignent de l'examen des inventions. Nous avons restreint notre étude aux ÉtatsUnis où le critère d'activité inventive fut " inventé » et où il fait partie intégrante de la culture légale liée à l'invention. De fait, l'immense majorité de la littérature relative au critère d'activité inventive ou à l'homme du métier est nord-américaine. Puisque les données sur l'évolution des pratiques des examinateurs de brevets eux-mêmes sont extrêmement restreintes (notamment au cours du $\mathrm{XIX}^{\mathrm{e}}$ siècle $)^{24}$, notre analyse se portera sur les raisonnements menés par les juges de la Cour suprême et des cours fédérales des États-Unis qui établissent la doctrine relative à l'interprétation de la loi. Cette approche correspond à la méthodologie usuelle de la littérature juridique qui s'appuie généralement sur une analyse des textes des cours de justice. Nous nous intéressons particulièrement aux analyses techniques effectuées par les juges leur permettant de conclure à l'inventivité des solutions. L'étude très complète de ces textes réalisée par John F. Duffy (2007) ${ }^{25}$, complétée par les travaux d'Amy L. Landers $(2010)^{26}$, a permis de dégager des décisions de jurisprudence régulièrement analysées et citées par les commentateurs contemporains de la loi. Nous avons donc identifié 24 décisions de jurisprudence entre 1822 et 2007 (récupérées depuis le site courtlistener.com, voir tableau 1). Leur influence majeure sur la doctrine liée à l'activité inventive permet de produire une grille d'analyse robuste des évolutions jurisprudentielles. Paradoxalement, ces décisions de jurisprudence révèlent une histoire confidentielle de l'invention : loin des décisions célèbres ${ }^{27}$ et des inventions emblématiques correspondantes, les décisions commentées par les juristes traitent d'inventions techniques méconnues (machine de découpe de bois, système de fixation de poignées de portes, système de désintégration d'argile, système de contrôle thermique d'un allume-cigare, amortisseurs etc.) : la particularité de ces inventions réside précisément dans la difficulté qu'elles posent aux évaluateurs du point de vue des critères d'évaluation. Par ailleurs, outre la jurisprudence, des changements légaux et institutionnels ont également influencé l'évaluation des brevets. Étant donné que le système du droit américain (dit de « common law ») se construit majoritairement sur la jurisprudence, nous établirons donc le découpage temporel de nos modèles à partir des évolutions de la jurisprudence plutôt que des textes de loi comme les Patent Acts ou des évolutions institutionnelles qui ont touché le système des brevets.

Quel cadre d'analyse choisir alors pour étudier ces jugements ? Puisqu'il s'agit de rendre compte du raisonnement des juges lors de l'examen, et que ce modèle retrace, par nature, un raisonnement de conception (en comparant celui de l'homme du métier - fictif - et celui décrit par l'invention), nous emploierons la théorie $\mathrm{C} / \mathrm{K}$ (Concept) Knowledge $)^{28}$. Ce cadre théorique sera mobilisé pour analyser les raisonnements techniques des juges américains lors de l'évaluation des inventions. L'utilisation d'une théorie de la conception apparaît judicieuse car, dans le

\footnotetext{
${ }^{24}$ Les pratiques des examinateurs de brevets sont normées par le Manual of Patent Examining Procedure (https:// www.uspto.gov/web/offices/pac/mpep/index.html) (consulté le 11/07/2019); la version la plus ancienne et accessible est de 1948.

${ }^{25}$ J. F. Duffy, « Inventing Invention: a Case Study of Legal Innovation », art. cit.

${ }^{26}$ A. L. Landers, « Ordinary Creativity in Patent Law: The Artist Within the Scientist », art. cit.

${ }^{27}$ Par exemple, le cas des procès relatifs au télégraphe de Morse, la controverse entre Bell et Gray relative à la paternité du téléphone, ou encore plus récemment la décision Kodak v. Polaroid.

${ }^{28}$ A. Hatchuel et B. Weil, « C-K Design Theory: an Advanced Formulation », Research in Engineering Design, vol. 19, n 4, 2009, p. 181-192. Voir également la présentation de la théorie dans S. Lenfle et L. Petitgirard, «L'invention du transistor aux Bell Labs ou la création d'une expertise sur un domaine inconnu », Entreprises et Histoire, n 98 , avril 2020.
} 
cadre de l'évaluation de l'activité inventive, les juges évaluent l'activité de conception menée par l'inventeur en se plaçant dans un référentiel de connaissances donné. $\mathrm{La}$ théorie $\mathrm{C} / \mathrm{K}$ présente par ailleurs plusieurs avantages dans le cadre de notre analyse : elle est indépendante du type de connaissances mobilisées, ce qui permet d'analyser dans un cadre commun des raisonnements menés sur une diversité d'objets ; elle permet de rendre compte des voies de conception décrivant les types d'inventions analysés par les juges; et enfin l'existence de l'espace des connaissances apparaît particulièrement pertinente pour saisir la définition de l'homme du métier ${ }^{29}$.

La théorie de la conception $\mathrm{C} / \mathrm{K}$ offre un formalisme représentant les activités de conception comme des opérations entre deux espaces : un espace de concept (C) et un espace de connaissances $(\mathrm{K})$. L'espace des concepts renvoie aux propositions sans statut logique (on ne peut dire si elles sont vraies ou fausses) décrivant les objets que l'on désire concevoir. L'espace des connaissances renvoie quant à lui à l'ensemble des propositions ayant un statut logique (connaissances scientifiques, techniques, industrielles, savoir-faire etc., pour lesquelles on peut dire si elles sont vraies ou fausses). L'activité de conception est alors modélisée au travers de quatre opérateurs : $\mathrm{K} \rightarrow \mathrm{C}$ (des connaissances sont utilisées pour ajouter des propriétés à l'objet à concevoir), $\mathrm{C} \rightarrow \mathrm{C}$ (les concepts peuvent être subdivisés en sous-concepts), $\mathrm{C} \rightarrow \mathrm{K}$ (les concepts suggèrent l'activation ou la création de connaissances), $\mathrm{K} \rightarrow \mathrm{K}$ (des connaissances peuvent être déduites de connaissances existantes au travers de règles logiques, de tests, d'apprentissages divers). Par ailleurs, certaines recherches ont récemment proposé une théorie de l'invention dont on connaît mieux les conditions formelles. Le modèle suivant décrit ainsi formellement ce que serait une « invention $»^{30}$ :

1) Considérons l'état de l'art accessible à l'homme du métier et connu de lui (auquel on intègre également les connaissances communes liées au domaine d'activité pertinent pour l'invention), que l'on note K(PSA) (Person Skilled in the Art, désormais PSA). Cette connaissance permet alors à un homme du métier de générer un ensemble de concepts, i.e. de nouveaux objets par modification, altération de propriétés mais qui n'exigent pas l'emploi de connaissances autres que celles d'un homme du métier (Espace K : K(PSA)).

2) Dans ce contexte, un concept inventif peut être théorisé comme une partition dite " expansive ${ }^{31}$, i.e. la génération d'un concept dont la connaissance associée (notée $\Delta K$ ) est non-atteignable par l'homme du métier : cette connaissance est dite « indépendante de $\mathrm{K}(\mathrm{PSA})$ » mais elle est nécessaire pour atteindre le concept inventif proposé. Cette indépendance des bases de connaissances a été théorisée sous le terme de $«$ splitting conditions $\rangle^{32}$.

Ce modèle met l'accent sur une définition de l'invention qui reposerait non sur un acte « créatif » mais bien plutôt sur un rapport d'indépendance entre les connaissances existantes (et potentielles) de l'homme du métier et celles apportées par l'invention. Cette indépendance n'est pourtant pas simple à décrire puisqu'elle suppose d'être capable de délimiter ce que sont les « connaissances potentielles »d'un homme du métier,

\footnotetext{
${ }^{29}$ A. Hatchuel, P. Le Masson, Y. Reich et E. Subrahmanian, « Design Theory: a Foundation of a New Paradigm for Design Science and Engineering ", Research in Engineering Design, vol. 29, n 1, 2018, p. 5-21 ; P. Le Masson et B. Weil, «La conception innovante comme mode d'extension et de régénération de la conception réglée : les expériences oubliées aux origines des bureaux d'études », Entreprises et Histoire, n 58, avril 2010, p. 51-73.

${ }^{30}$ O. Kokshagina, P. Le Masson et B. Weil, « Should we Manage the Process of Inventing? Designing for Patentability », Research in Engineering Design, vol. 28, n 4, 2017, p. 457-475.

${ }^{31}$ A. Hatchuel et B. Weil, « A New Approach of Innovative Design: an Introduction to C-K Theory », DS 31: Proceedings of ICED 03, the 14th International Conference on Engineering Design, Stockholm, 2003.

${ }^{32}$ P. Le Masson, A. Hatchuel, et B. Weil, « Design Theory at Bauhaus: Teaching "Splitting” Knowledge », Research in Engineering Design, vol. 27, $\mathrm{n}^{\circ}$ 2, 2016, p. 91-115.
} 


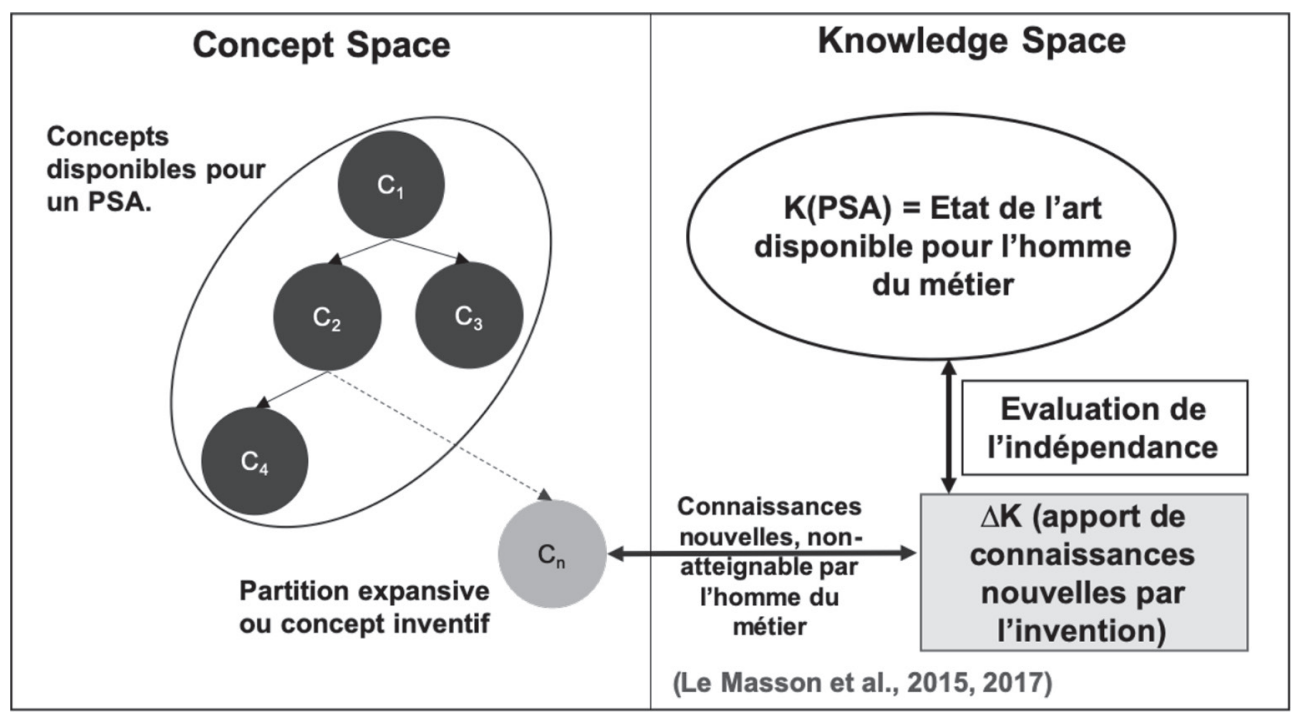

Figure 1. Modèle de I'invention dans la théorie $\mathrm{C} / \mathrm{K}$ appliqué à l'évaluation du critère d'activité inventive

c'est-à-dire les tests, expérimentations et modifications qu'il est capable lui-même d'effectuer sans avoir à être « inventif » mais qui produisent tout de même de nouvelles connaissances. C'est bien cette frontière énigmatique que le droit du brevet cherche à tracer.

Cette représentation formelle de l'invention repose donc sur l'établissement d'un double régime : un régime de représentation et de description de l'état de l'art particulier que nous appellerons « structure de l'état de l'art » pour indiquer un ordonnancement particulier des éléments de connaissances ; et, deuxièmement, un régime de preuve d'indépendance, construit simultanément avec le premier régime, qui fournit les règles permettant d'établir si deux éléments de connaissances sont «indépendants ». On comprend alors que caractériser une évaluation de l'invention va nécessairement impliquer trois observations :

1) L'observation de l'état de l'art pris comme connaissances disponibles pour l'homme du métier - K(PSA) : quel est l'état de l'art mobilisé par les juges pour décrire les connaissances de l'homme du métier? quelle est la structure de cet état de l'art?

2) L'observation de l'apport de l'invention - $\Delta \mathrm{K}$ : quel est l'apport de la solution technique évaluée par rapport à l'état de l'art ? quelle est la nouveauté introduite par le brevet?

3) L'observation du critère d'indépendance : comment le juge établit-il que la nouvelle connaissance apportée par l'invention n'était pas « atteignable » par un homme du métier?

En focalisant notre attention sur le critère d'activité inventive, nous avons donc sélectionné des décisions dans lesquelles la nouveauté de l'invention est relativement admise : comme nous le verrons, le critère d'activité inventive émerge précisément à cause de questions portant sur différents degrés de nouveauté. Par ailleurs, certains critères comme l'application industrielle (l'utilité), la suffisance de description ou l'unité d'invention ne seront pas ou peu commentés.

L'analyse historique sera donc présentée en deux temps : premièrement, nous décrirons le 


\begin{tabular}{|c|c|}
\hline Date & Décisions de jurisprudence étudiées \\
\hline 1822 & Evans v. Eaton, 7 Wheat. 356, 5 L. Ed. 472; 1822 U.S. LEXIS 266 \\
\hline 1825 & Earle v. Sawyer, 8 F. Cas. 254 (C.C.D. Mass. 1825) \\
\hline 1851 & Hotchkiss v. Greenwood, 52 U.S. 248, 13 L. Ed. 683, 11 How. 248, 1850 U.S. LEXIS 1507 \\
\hline 1870 & Stimpson v. Woodman, 77 U.S. 117, 19 L. Ed. 866, 10 Wall. 117, 1869 U.S. LEXIS 1048 \\
\hline 1874 & Rubber-Tip Pencil Co. v. Howard, 87 U.S. 498, 22 L. Ed. 410, 20 Wall. 498, 1874 U.S. LEXIS 1439 \\
\hline 1874 & Hailes v. Von Wormer, 87 U.S. 353, 22 L. Ed. 241, 20 Wall. 353, 1873 U.S. LEXIS 1509 \\
\hline 1876 & Reckendorfer v. Faber, 92 U.S. 347, 23 L. Ed. 719, 1875 U.S. LEXIS 1765 \\
\hline 1883 & Atlantic Works v. Brady, 107 U.S. 192, 2 S. Ct. 225, 27 L. Ed. 438, 1882 U.S. LEXIS 1215 \\
\hline 1888 & $\begin{array}{l}\text { Gosnell v. Bishop, Reports of Patent, Design and Trade Mark Cases, Volume 5, Issue 6, } 31 \text { March } \\
\text { 1888, Pages 151-159 }\end{array}$ \\
\hline 1895 & Potts v. Creager, 155 U.S. 597, 15 S. Ct. 194, 39 L. Ed. 275, 1895 U.S. LEXIS 2109 \\
\hline 1908 & $\begin{array}{l}\text { Continental Paper Bag Co. v. Easter Paper Bag Co., } 210 \text { U.S. 405, } 28 \text { S. Ct. 748, } 52 \text { L. Ed. 1122, } \\
1908 \text { U.S. LEXIS } 1519\end{array}$ \\
\hline 1938 & $\begin{array}{l}\text { Lincoln Engineering Co v. Stewart-Warner Corp, } 303 \text { U.S. 545, } 58 \text { S. Ct. 662, } 82 \text { L. Ed. 1008, } \\
1938 \text { U.S. LEXIS } 391\end{array}$ \\
\hline 1941 & Cuno Engineering v. Automatic Corp, 314 U.S. 84, 62 S. Ct. 37, 86 L. Ed. 58, 1941 U.S. LEXIS 1250 \\
\hline 1949 & $\begin{array}{l}\text { Jungersen v. Ostby \& Barton Co., } 335 \text { U.S. 560, } 69 \text { S. Ct. 269, } 93 \text { L. Ed. 2d 235, } 1949 \text { U.S. } \\
\text { LEXIS } 3052\end{array}$ \\
\hline 1950 & Graver Mfg Co. v. Linde Co., 339 U.S. 605, 70 S. Ct. 854, 94 L. Ed. 2d 1097, 1950 U.S. LEXIS 2608 \\
\hline 1951 & $\begin{array}{l}\text { A \& P Co. Tea v. Supermarket Corp., } 340 \text { U.S. 147, } 71 \text { S. Ct. 127, } 95 \text { L. Ed. 2d 162, } 1950 \text { U.S. } \\
\text { LEXIS } 2604\end{array}$ \\
\hline 1966 & $\begin{array}{l}\text { Graham v. John Deere Co. of Kansas City, } 383 \text { U.S. 1, } 86 \text { S. Ct. 684, } 15 \text { L. Ed. 2d 545, } 1966 \text { U.S. } \\
\text { LEXIS } 2908\end{array}$ \\
\hline 1966 & United States v. Adams, 383 U.S. 39, 86 S. Ct. 708, 15 L. Ed. 2d 572, 1966 U.S. LEXIS 2754 \\
\hline 1969 & $\begin{array}{l}\text { Anderson's Black Rock v. Pavement Salvage, } 396 \text { U.S. 57, } 90 \text { S. Ct. 305, } 24 \text { L. Ed. 2d 258, } 1969 \\
\text { U.S. LEXIS } 3322\end{array}$ \\
\hline 1976 & Sakraida v. Ag Pro, Inc., 425 U.S. 273, 96 S. Ct. 1532, 47 L. Ed. 2d 784, 1976 U.S. LEXIS 146 \\
\hline 1985 & Interconnect Planning Corporation v. Feil, 774 F.2d 1132 \\
\hline 2006 & In Re Kahn, 441 F.3d 977 \\
\hline 2007 & KSR Co. v. Teleflex, 550 U.S. 398, 127 S. Ct. 1727, 167 L. Ed. 2d 705, 2007 U.S. LEXIS 4745 \\
\hline
\end{tabular}

\section{Tableau 1. Décisions de jurisprudence étudiées}

cadre légal et institutionnel qui sert de contexte aux différents régimes ; deuxièmement, nous analyserons plusieurs décisions de jurisprudence considérées par les juristes comme des points de référence jurisprudentiels concernant la variété des doctrines adoptées. La première décision, Evans v. Eaton (1822), nous permettra d'établir le modèle initial tel qu'il est adopté par les tribunaux au début du XIX ${ }^{\mathrm{e}}$ siècle. La décision Hotchkiss v. Greenwood (1851) est particulièrement célèbre car les juges y introduisent une figure d'homme du métier comme référentiel de connaissances. Quatre-vingt-dix ans plus tard, la décision Cuno Engineering Corp. v. Automatic Corp (1941) rompt avec les interprétations jurisprudentielles précédentes en reconnaissant l'existence du critère d'activité inventive comme un critère « autre » qui n'est plus une extension du 
critère de nouveauté mais correspond bien à une évaluation d'un autre ordre. Enfin, nous présenterons Graham v. John Deere (1966), jugement dans lequel le tribunal introduit pour la première fois un processus explicite associé à l'évaluation du critère d'activité inventive. Ce sont donc ces analyses, couplées à l'étude de la littérature juridique, qui nous permettent d'établir trois modèles correspondant à chaque régime d'invention. Nous conclurons par l'analyse de la décision KSR v. Teleflex (2007) qui marque, d'après la littérature juridique, une nouvelle rupture dans les pratiques d'évaluation. Afin de valider la cohérence de nos modèles, nous utiliserons les 19 autres décisions de jurisprudence comme cas de validation : celle-ci sera fondée sur les deux variables décrites précédemment (structure de l'état de l'art et critère d'indépendance).

\section{LE RÉGIME DE LA NOUVEAUTÉ (1790-1851) : LES DÉBUTS DU SYSTÈME AMÉRICAIN DES BREVETS}

Le 10 avril 1790, les États-Unis adoptent "An Act to promote the progress of useful Arts » posant les premières pierres du système américain des brevets et statuant qu'une lettre de patente ne peut être attribuée à un inventeur que pour les objets alors décrits ainsi : «any useful art, manufacture, engine, machine, or device, or any improvement therein not before known or used $\gg{ }^{33}$.

Cette liste se voit complétée par des critères laissés à l'appréciation d'examinateurs qui doivent déterminer si l'invention est suffisamment utile et importante. Ce texte spécifie donc d'une part les types d'objets susceptibles d'être brevetés et d'autre part une condition essentielle à la brevetabilité : l'utilité de l'art produit (« any useful art»). En 1793, le critère de nouveauté apparaît explicitement (" any new and useful art, machine, manufacture or composition of matter »), et enfin une nouvelle condition plus restrictive apparaît sur la nature de l'invention : "And it is hereby enacted and declared, that simply changing the form or the proportions of any machine, or composition of matter, in any degree, shall not be deemed a discovery $»^{34}$.

On considère alors que la modification des objets se réduisant à une transformation géométrique ne peut donner lieu à un brevet. L'ajout de cette règle n'est pas anecdotique car elle introduit déjà un principe d'évaluation de la nouveauté. Par ailleurs, il est fortement probable que le secrétaire d'État, le secrétaire à la Défense et le procureur général alors chargés de l'examen aient été confrontés à ce type d'inventions de formes. On peut noter que la France introduit en 1791 une règle de nature similaire qui inspire sans doute Thomas Jefferson $^{35}$, en grande partie à l'origine de la rédaction du Patent Act de 1793. L'examen dit préalable (avant la délivrance du titre) étant considéré trop long et coûteux, de 1793 à 1836 les inventions sont enregistrées sur la base de la bonne foi des inventeurs. Ce sont les tribunaux qui sont alors garants du respect des critères de brevetabilité (nouveauté et utilité) et ceux-ci s'appuient essentiellement sur les éléments fournis par les deux parties opposées. D’autres éléments viennent cependant compléter l'analyse des juges et en particulier la doctrine anglaise continue d'alimenter les décisions des tribunaux américains (voir à titre d'exemple la décision rendue dans le procès Earle v. Sawyer (1825) citant la décision Brunton v. Hawkes ${ }^{36}$ ). Il faut alors noter que les tribunaux anglais ont conservé une interprétation stricte de la nouveauté, c'est-à-dire que toute altération d'une technique qui est issue d'un procédé

\footnotetext{
${ }^{33}$ Patent Act, « An Act to Promote the Progress of Useful Arts », op. cit.

${ }^{34}$ Patent Act of 1793, Ch. 11, 1 Stat. 318-323, 21 février 1793.

${ }^{35}$ T. Jefferson, « Draft of a Bill to Promote the Progress of the Useful Arts », 7 février 1791.

${ }^{36}$ W. Carpmael, Law Reports of Patent Cases, op. cit., p. 405.
} 
ou d'une propriété nouvelle est reconnue comme invention; en particulier, l'utilité (qui comprend par exemple la baisse de prix ou la facilité d'usage) apparaît comme un argument clé $^{37}$. Cependant les tribunaux américains prennent rapidement leurs distances avec cette doctrine, comme en témoignent les décisions suivantes.

En 1822 se tient un procès opposant Evans à Eaton ${ }^{38}$. Le plaignant, Evans, intente une action en contrefaçon contre Eaton concernant une machine employée dans la production de farine appelée le Hopperboy. L'objectif de cette machine est de répandre une substance granuleuse (farine) sur le sol, de la mélanger par l'action d'un bras muni d'éléments de convoyage pour la sécher et la refroidir tout en amenant la farine vers le centre pour pouvoir la stocker dans des conteneurs. La machine est actionnée grâce à de l'énergie hydraulique et consiste en un axe de forme ronde et de deux bras (l'un au-dessus, l'un en dessous). Le bras proche du sol à hauteur variable est entraîné dans un mouvement circulaire par l'action des cordes qui le rattachent au bras supérieur tout en exerçant, grâce à un poids, une tension maintenant le bras inférieur horizontal (voir figure 2).

La défense de Eaton cherche à prouver qu'Evans n'est pas l'inventeur originel du Hopperboy. Le jugement traite donc bien de la nouveauté de l'invention brevetée par Evans. Le jugement prononcé par le juge Story permet alors de repérer les trois observations requises dans le cadre de notre analyse :

\section{1) État de l'art - établissement et sélection des antériorités relatives à l'invention : un ensemble de témoins présentés par les différentes parties est mobilisé pour prou- ver l'utilisation de Hopperboys similaires à}

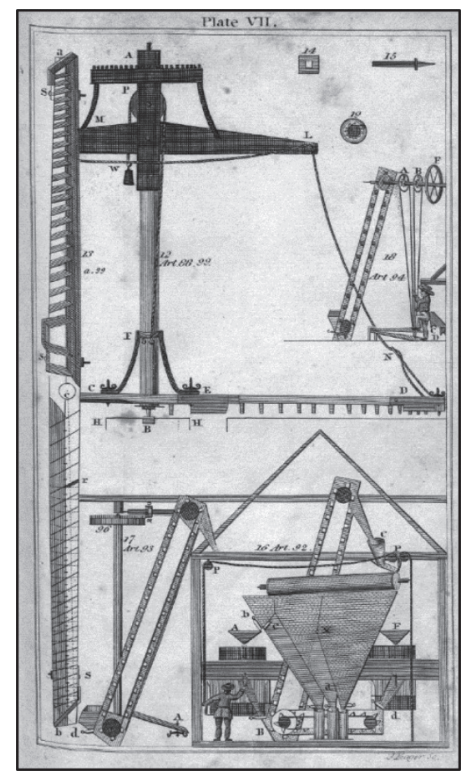

Figure 2. Le Hopperboy d'Evans ${ }^{39}$

une date antérieure à la date d'application du brevet.

«Daniel Stouffer, who deposes, that he first saw the Stouffer Hopperboy in his father's, Christian Stouffer's mill, in the year 1764. In the year 1775 or 1776, he erected a similar one, in the mill of his brother Henry, and another in Jacob Stouffer's mill, in 1777, 1778, or $1779 »^{40}$.

2) Apport de l'invention - analyse des différences entre les revendications du brevet (Hopperboy d'Evans) et une unique invention pertinente de l'état de l'art (Hopperboy de Stouffer): ces deux inventions sont comparées élément par élément.

«In what do they differ? The plaintiff's shaft is round, and consequently could not turn the

\footnotetext{
${ }^{37}$ Cf. Crane v. Price, Jugement du 13 juin 1842 par le juge N. C. Tindal, in T. Webster, Reports and Notes of Cases on Letters Patent for Inventions, Londres, Thomas Blenkarn, 1844, p. 407-417 : "The only question, therefore, that ought to be considered on the evidence is, was the iron produced by the combination of the hot blast and the anthracite a better or a cheaper article than was before produced from the combination of the hot blast and the bituminous coal; and was the combination, described in the specification, new as to the public use thereof in England 》.

${ }^{38}$ Evans v. Eaton, 7 Wheat. 356, 5 L. Ed. 472; 1822 U.S. LEXIS 266.

39 O. Evans, « The Young Mill-Wright and Miller's Guide », Lea \& Blanchard, 1848, Figure 12, plate VII.

${ }^{40}$ Evans v. Eaton, op. cit.
} 
arm, into which it is loosely inserted, if it were not for the cords which connect the extremities of the arm to those of the leader. The shaft of the Stouffer Hopperboy is square, and therefore turns the arm without the aid of a leader or of cords ${ }^{41}$

\section{3) Critère d'indépendance - analyse} de la nouveauté : étant donné les différences structurelles entre le « Stouffer Hopperboy» et le « Evans Hopperboy », doit-on reconnaître le dernier comme un objet nouveau (et donc reconnaître Evans inventeur d'une machine qui n'existait pas précédemment) ou comme une simple amélioration du Stouffer Hopperboy (et donc décider qu'Evans ne peut que prétendre à un brevet de perfectionnement) ? La réponse du juge est alors la suivante :

"I take the rule to be, and so it has been settled in this, and in other Courts, that if the two machines be substantially the same, and operate in the same manner, to produce the same result, though they may differ in form, proportions, and utility, they are the same in principle; and the one last discovered has no other merit than that of being an improved imitation of the one before discovered and in use, for which no valid patent can be granted $»^{42}$.

Le juge Story insiste sur le fait qu'une invention ne peut être attribuée à un concepteur que si « elle est substantiellement nouvelle dans sa structure et son mode opératoire ${ }^{43}$. Le juge défend aussi que s'il est admis qu'un brevet ne constitue qu'une amélioration par rapport à l'état de l'art existant, le droit qu'il confère se réduit alors à cette dite amélioration. On constate également que le juge explicite la nature des dimensions de l'objet à partir duquel les différences entre deux brevets pourront être établies. Si la Cour suprême accepte les propriétés liées à l'opération de la machine ou la production d'un résultat ("operate in the same manner, to produce the same result »), elle écarte d'autres éléments de distinction ( form, proportions and utility »). L'analyse de la différence entre deux objets et la qualité de cette différence s'appuie donc sur la mise en place d'un cadre de référence qui renvoie à des dimensions spécifiques des objets qui précisément rendront visibles les différences techniques. Ici, l'état de l'art mobilisé se manifeste au travers d'une machine, le Stouffer Hopperboy, à partir de laquelle les différences sont établies. C'est donc bien l'objet complet, pris comme référentiel, sur lequel s'appuie le jugement, même si cet objet est rapidement réduit à quelques dimensions suffisantes à l'analyse de la différence. Cette décision illustre deux points cruciaux : premièrement, l'état de l'art se représente alors par objet (ou ici par une " machine ») souvent pris individuellement et réduit à des dimensions spécifiques ; deuxièmement, le mode de description des objets s'attache aux propriétés qui les différencient.

Un deuxième jugement, prononcé en 1825, se caractérise par une structure similaire (Earle v. Sawyer, 1825) : le plaignant, Earle, intente une action en contrefaçon contre Sawyer pour la reproduction de sa machine à découpe de bardeaux de bois. Le brevet de Earle porte sur l'amélioration de cette machine par l'introduction d'une scie circulaire en remplacement de la scie perpendiculaire auparavant utilisée dans ce type de machines et par la modification des éléments nécessaires à l'introduction de ce nouveau mode de découpe dans la machine. Le brevet porte seulement sur l'amélioration apportée et correspond donc à un brevet de perfectionnement. La question soulevée devant le tribunal est celle de la nouveauté

\footnotetext{
${ }^{41}$ Ibid.

${ }^{42}$ Ibid.

${ }^{43}$ Ibid. D'après le juge Story : « the party can maintain a title to it only by establishing that it is substantially new in its structure and mode of operation $»$.
} 
de l'amélioration étant donné que les scies circulaires étaient déjà connues. La principale antériorité considérée est alors la machine de Sawyer. Après une description de l'invention, et de son apport par rapport à la machine de Sawyer (introduction de la scie circulaire), il est établi que l'amélioration à la date de son invention était bien nouvelle («There was no evidence in the case to show, that any person had ever, before the plaintiff's asserted invention, applied a circular saw in any manner to the plaintiff's old machine. »). Le raisonnement explicité est bien le même que celui du jugement précédent : l'état de l'art est réduit à un objet complet, et la distinction est établie à partir d'une propriété particulière (nature de la scie et mode opératoire). Cependant le jugement accentue une interprétation stricte du critère de nouveauté établissant que toute combinaison nouvelle d'éléments pourtant déjà existants permettant un perfectionnement est brevetable :

«That if the plaintiff were the first to apply or combine a circular saw with his original shingle mill for the purpose of making shingles, although the shingle mill were in common use, and the circular saw were in use (meaning, I presume, separately, and not in combination), and there were nothing new in the mode or machinery, by which it was applied (but meaning, I presume, that the combination itself was new), still the plaintiff is entitled to a patent $»^{44}$.

Du point de vue de l'évaluation des brevets, la seule condition est donc bien la nouveauté de la combinaison des éléments techniques, sans condition concernant la nature de la combinaison ou son effet. Précisons que le langage est ici plus tranché que dans la décision précédente : la question se réduit ici à l'existence d'un droit de propriété sur un perfectionnement; de fait, il est donc conclu que tout perfectionnement nouveau mérite un brevet. La décision insiste d'ailleurs sur le fait que la délivrance d'un brevet n'est nullement assujettie à une dimension potentiellement « inventive » de la solution technique :

«The whole argument, upon which this doctrine is attempted to be sustained, is, if I rightly comprehend it, to this effect. It is not sufficient, that a thing is new and useful, to entitle the author of it to a patent. He must do more. [...] If the result of accident, it must be what would not occur to all persons skilled in the art, who wished to produce the same result. [...] It did not appear to me at the trial, and does not appear to me now, that this mode of reasoning upon the metaphysical nature, or the abstract definition of an invention, can justly be applied to cases under the Patent Act. [...] The law looks to the fact, and not to the process by which it is accomplished $\gg{ }^{45}$.

Cette citation surprend : elle montre que dès 1825 l'avocat de Earle utilise déjà un raisonnement qui sera finalement adopté par les tribunaux vingt-cinq ans plus tard ; mais surtout l'interprétation du juge est toute autre puisqu'il condamne explicitement l'existence présupposée d'une définition, qualifiée d'abstraite, de l'invention qui pousserait à regarder le processus qui a mené à l'invention, plutôt que le résultat qui en découle. Avec cette décision, le juge Story entérine donc un critère d'évaluation du brevet (critère d'indépendance) qui peut être décrit comme un principe de nonidentité. Si l'invention décrit l'existence de propriétés, de structures, de modes opératoires nouveaux (qui n'étaient pas connus auparavant), alors elle est susceptible d'être brevetée, indépendamment de toute autre considération. L'évaluation d'une invention au regard des connaissances ou du savoir-faire d'un homme du métier est alors considérée en dehors de la loi. Nous résumons cette analyse dans le tableau 2.

Nous désignons donc la période 17901851 comme le régime de la nouveauté. Notons que ce régime est tout de suite peu stable et oblige les juges à une interprétation extrêmement stricte de ce qui fait nouveauté.

\footnotetext{
${ }^{44}$ Earle v. Sawyer, 8 F. Cas. 254 (C.C.D. Mass. 1825), décision rendue par le juge Story. ${ }^{45}$ Ibid.
} 


\begin{tabular}{|c|c|c|c|c|c|}
\hline $\begin{array}{c}\text { Décisions de } \\
\text { jurisprudence } \\
1790-1851 \\
\text { Régime de } \\
\text { la nouveauté }\end{array}$ & $\begin{array}{l}\text { Invention } \\
\text { mise en } \\
\text { cause }\end{array}$ & État de l'art & $\begin{array}{c}\text { Structure } \\
\text { d'état de } \\
\text { l'art }\end{array}$ & $\begin{array}{l}\text { Apport de } \\
\text { l'invention }\end{array}$ & $\begin{array}{c}\text { Critère } \\
\text { d'indépen- } \\
\text { dance }\end{array}$ \\
\hline $\begin{array}{l}\text { Evans v. Eaton } \\
\text { (1822) }\end{array}$ & $\begin{array}{l}\text { Hopperboy - } \\
\text { machine de } \\
\text { production de } \\
\text { farine }\end{array}$ & $\begin{array}{l}\text { Stouffer } \\
\text { Hopperboy }\end{array}$ & $\begin{array}{l}\text { Unique } \\
\text { antériorité } \\
\text { de type objet }\end{array}$ & $\begin{array}{l}\text { Ajout d'un } \\
\text { bras } \\
\text { supérieur avec } \\
\text { système de } \\
\text { contrepoids }\end{array}$ & $\begin{array}{l}\text { Perfection- } \\
\text { nement non } \\
\text { connu dans } \\
\text { l'état de l'art }\end{array}$ \\
\hline $\begin{array}{l}\text { Earle v. } \\
\text { Sawyer } \\
\text { (1825) }\end{array}$ & $\begin{array}{l}\text { "Shingle } \\
\text { mills»- } \\
\text { machine de } \\
\text { découpe de } \\
\text { bardeaux de } \\
\text { bois à } \\
\text { scie circulaire }\end{array}$ & $\begin{array}{l}\text { Shingle mills } \\
\text { à scie } \\
\text { perpendiculaire }\end{array}$ & $\begin{array}{l}\text { Unique } \\
\text { antériorité } \\
\text { de type objet }\end{array}$ & $\begin{array}{l}\text { Substitution } \\
\text { de la scie } \\
\text { perpendiculaire } \\
\text { par une scie } \\
\text { circulaire dans } \\
\text { la machine de } \\
\text { découpe }\end{array}$ & $\begin{array}{l}\text { Substitution } \\
\text { non connue } \\
\text { dans l'état } \\
\text { de l'art }\end{array}$ \\
\hline
\end{tabular}

Tableau 2. Description de deux décisions de jurisprudence pour le régime de la nouveauté (1790-1851)

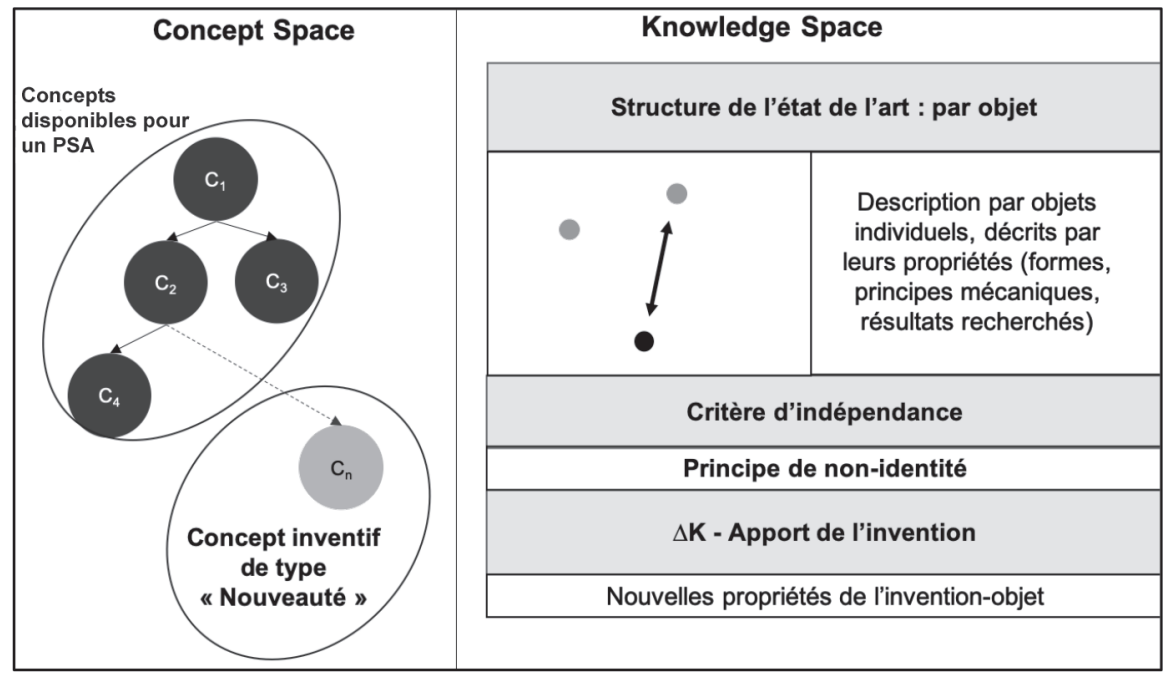

Figure 3. Synthèse du modèle d'invention pour le régime de la nouveauté (1790 - 1851) interprété $\mathrm{d}^{\prime}$ après la théorie $\mathrm{C} / \mathrm{K}$.

Étant donné le manque de robustesse du critère de nouveauté, le critère d'utilité occupe en fait une place importante et pousse les juges à trancher en faveur de l'invention « utile». Cependant, dès 1851, cette interprétation atteint ses limites et provoque un changement de paradigme.
LE RÉGIME DU PROGRÈS FONCTIONNEL (1851 - 1940) : L'ÉMERGENCE DU CRITÈRE D'ACTIVITÉ INVENTIVE

Le Patent Act de 1836 réintroduit l'examen préalable. C'est à cette occasion que sont 
créés aux États-Unis l'office des brevets et la fonction d'examinateur de brevets telle que nous la connaissons aujourd'hui ${ }^{46}$. Jusqu'alors, il n'existait donc pas de recherche dans l'état de l'art effectuée par l'administration américaine. La mise en place de l'office change l'approche : au-delà de la délivrance des titres, l'institution devient responsable de l'enregistrement, de la conservation et de la publication des brevets. Par la suite, le Patent Act de 1870 renforce la position de l'office des brevets en nommant 22 examinateurs principaux et en établissant que l'institution doit conserver tout document relatif aux brevets (enregistrements, livres, dessins, modèles, spécifications). L'état de l'art devient donc plus facile d'accès pour les examinateurs de brevets et commence à se structurer au travers de classifications. Cette structuration rend évidemment plus aisée la recherche dans l'état de l'art alors même que le nombre de brevets augmente, mais elle permet également une division de la charge de travail chez les examinateurs, orientant les différents brevets vers différents services au sein de l'office ${ }^{47}$. Les procès qui ont alors lieu ne reposent donc plus seulement sur une confrontation entre deux parties opposées qui chacune fait valoir ses arguments, en invoquant parfois certains experts puisque les examinateurs apparaissent désormais comme des tiers administratifs apportant de nouvelles connaissances aux juges. En 1851, la décision Hotchkiss v. Greenwood introduit pour la première fois la figure de l'homme du métier pour justifier l'absence de nouveauté dans certaines inventions ${ }^{48}$. Cette décision marque la naissance du critère d'activité inventive au sein du système américain des brevets, et tient à ce titre une place particulière dans son étude. En 1940, la décision Cuno Engineering
Corp v. Automatic Corp marque une nouvelle rupture jurisprudentielle en introduisant l'analyse de l'activité inventive comme une analyse distincte de la nouveauté. Nous décrirons plusieurs décisions plus en détail afin d'illustrer les transformations du raisonnement du juge : Hotchkiss v. Greenwood (1851) déjà évoquée, Reckendorfer v. Faber (1876), et Potts v. Creager (1895).

La première décision concerne un litige autour d'un brevet, déposé par Hotchkiss, à propos de l'amélioration des poignées (de portes ou autres) fabriquées à partir d'argile ou de porcelaine. Le brevet porte sur différents moyens d'attaches et procédés permettant la fixation de la poignée (en argile ou porcelaine) sur son support : ce procédé consiste à fixer une tige dans une cavité creusée dans la poignée puis à attacher par soudage une vis servant à la fixation (voir figure 4). La défense de Greenwood s'appuie sur la démonstration que ce brevet n'est pas original (« want of originality »), en montrant d'une part que le procédé d'attache revendiqué était déjà employé dans le cas d'une poignée en métal et d'autre part que la substitution de la porcelaine ou de l'argile au métal ou au bois n'est pas "inventive ». Ces éléments de contexte permettent alors d'introduire le jugement technique effectué par la Cour suprême des États-Unis :

1) État de l'art : L'état de l'art mobilisé concerne les poignées de portes, et notamment des moyens d'attaches (tige, broche) qui leur sont associés, l'état de l'art renvoie donc non plus à une unique antériorité mais à un ensemble de connaissances techniques associées à ce domaine :

«knobs of metal, wood, etc., connected with a shank and spindle, in the mode and

\footnotetext{
${ }^{46}$ K.W. Swanson, « The Emergence of the Professional Patent Practitioner », art. cit.

${ }^{47}$ F. Lafond et D. Kim, « Long-run Dynamics of the US Patent Classification System », Journal of Evolutionary Economics, vol. 29, n 2, 2019, p. 631-664.

48 À noter que la figure d'homme du métier à proprement parler existe dès le Patent Act de 1790 (cf. Section 2 : « And be it further enacted, That the grantee or grantees of each patent shall [...] deliver to the Secretary of State a specification in writing, containing a description $[. .$.$] ; which specification shall be so particular [. .$.$] to enable a$ workman or other person skilled in the art or manufacture [...] to make, construct, or use the same [...] »). L'emploi de cette figure pour contrôler la suffisance de description n'est pas nouveau mais c'est son usage dans le cadre de l'évaluation de la nouveauté qui fait l'originalité de la décision Hotchkiss v. Greenwood.
} 
by the means used by the patentees in their manufacture, had been before known, and were in public use at the date of the patent ${ }^{49}$.

2) Apport de l'invention : La différence entre le brevet de Hotchkiss et l'état de l'art est résumée comme suit :

"...hence the only novelty which could be claimed on their part was the adaptation of this old contrivance to knobs of potter's clay or porcelain; in other words, the novelty consisted in the substitution of the clay knob in the place of one made of metal or wood $»^{50}$.

Notons dans ce cas précis que les poignées en porcelaine étaient connues et que c'est donc l'adaptation du système de fixation à ces poignées en porcelaine qui est l'objet du brevet. Formellement, cette combinaison est bien nouvelle.

3) Critère d'indépendance : Cette fois-ci le jugement ne reconnaît pas ce type de combinaisons comme brevetable :

«Now it may very well be, that, by connecting the clay or porcelain knob with the metallic shank in this well-known mode, an article is produced better and cheaper than in the case of the metallic or wood knob; but this does not result from any new mechanical device or contrivance [...]. The improvement consists in the superiority of the material, and which is not new, over that previously employed in making the knob $»^{51}$.

La nouveauté de l'invention de combinaison ne peut être établie que si la combinaison provoque la création d'un nouveau moyen ("new mechanical device or contrivance ») ou qu'elle permet d'obtenir un effet supérieur ( $"$ the superiority of the material, which is not new»). Par ailleurs, ici l'apport positif de la combinaison («better and cheaper ») n'est pas considéré comme

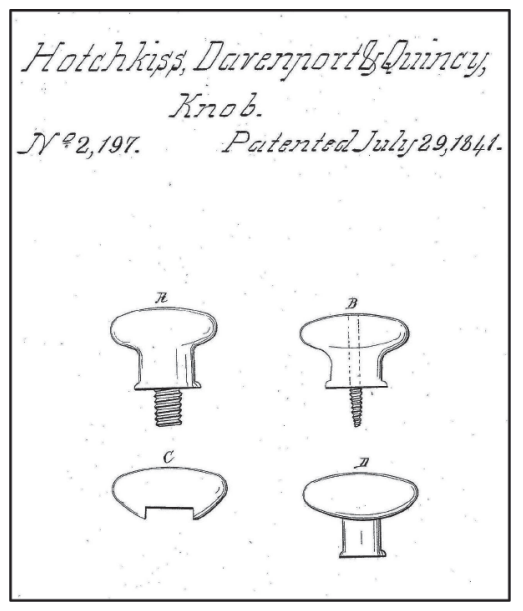

Figure 4. Poignées de porte par Hotchkiss ${\text { (Patent } N^{\circ} 2197,29 \text { juillet 1941) }}^{52}$

un effet technique dérivant de l'invention. Le juge généralise ensuite son propos :

"...for unless more ingenuity and skill in applying the old method of fastening the shank and the knob were required in the application of it to the clay or porcelain knob than were possessed by an ordinary mechanic acquainted with the business, there was an absence of that degree of skill and ingenuity which constitute essential elements of every invention. In other words, the improvement is the work of the skillful mechanic, not that of the inventor ${ }^{53}$.

Le juge introduit une nouvelle forme de connaissances dans l'état de l'art : l'ingéniosité et la compétence d'un mécanicien connaissant l'industrie ; et il établit qu'une invention doit précisément dépasser cette connaissance. Par opposition, toute solution technique échouant à cet exercice de pensée est assimilée à une amélioration non brevetable. La représentation de l'invention diffère donc largement de celle du modèle précédent en introduisant deux conditions : l'une étant que la combinaison ne

\footnotetext{
${ }^{49}$ Hotchkiss v. Greenwood, op. cit.

${ }^{50}$ Ibid.

${ }^{51} \mathrm{Ibid}$

${ }^{52}$ Extrait de USPTO, patent search PatFT : http://patft.uspto.gov.

${ }^{53}$ Hotchkiss v. Greenwood, op. cit.
} 


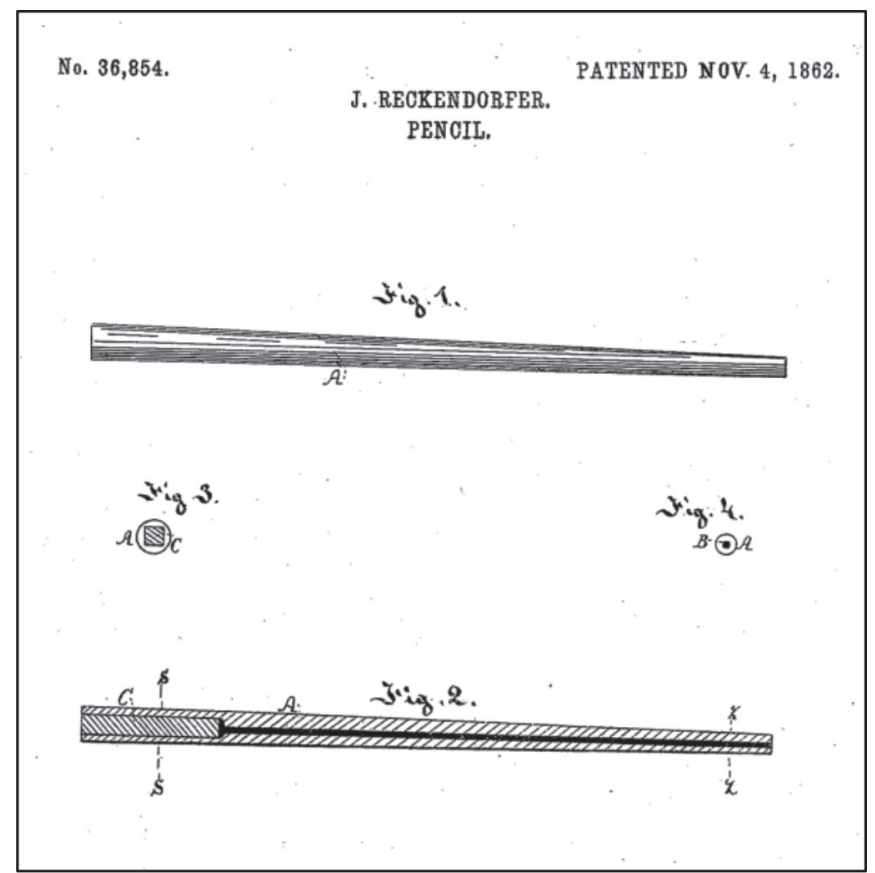

Figure 5. Crayon à papier par J. Reckendorfer (Patent $\mathrm{N}^{\circ} 36$ 854, 4 novembre 1862)

peut être un simple agrégat d'éléments connus mais doit nécessiter a minima la création d'une nouvelle action ou d'un nouvel effet pour la permettre ; l'autre étant que la capacité à combiner des éléments connus d'un même domaine technique en vue d'une amélioration est assimilée à une compétence de l'homme du métier. L'état de l'art mobilisé diffère en fait également en nature par rapport au régime présenté précédemment : il n'est plus question d'un objet unique complet mais d'une catégorie d'objets techniques, les poignées de porte (bien que la description en soit ici laconique). Le critère d'indépendance appelle quant à lui quelques commentaires : au-delà du discours relatif au " mécanicien ordinaire », le juge évalue la qualité de l'amélioration apportée par la substitution du matériau. Il constate alors et admet que la poignée puisse être moins chère et meilleure en tout point, mais cela est indépendant de l'objet auquel il s'applique puisqu'aucune autre modification n'a dû être apportée. Il $\mathrm{y}$ a donc un raisonnement sur la nature de l'amélioration apportée : si elle n'implique pas une modification de certaines propriétés particulières des systèmes de fixation de la poignée de porte, elle est alors admise comme évidente pour un homme du métier.

Ces particularités du raisonnement se répliquent alors dans d'autres décisions célèbres, tout en s'affinant. En 1876, Joseph Reckendorfer intente un procès pour violation de son brevet portant sur un crayon à papier, disposant d'une extrémité élargie avec une cavité pouvant accueillir un effaceur ou tout autre objet utile, et dont la structure en bois s'affine graduellement sur toute la longueur du crayon depuis la partie supérieure jusqu'à la pointe (cf. figure 5) pour une meilleure préhension. Dans ce cas précis, l'invention porte sur l'adaptation de la forme du crayon pour permettre une combinaison du crayon avec une pièce de caoutchouc servant d'effaceur. De nouveau, les effaceurs de caoutchouc étaient connus et le crayon également, cependant la combinaison, bien que nouvelle, n'est pas admise comme invention : 

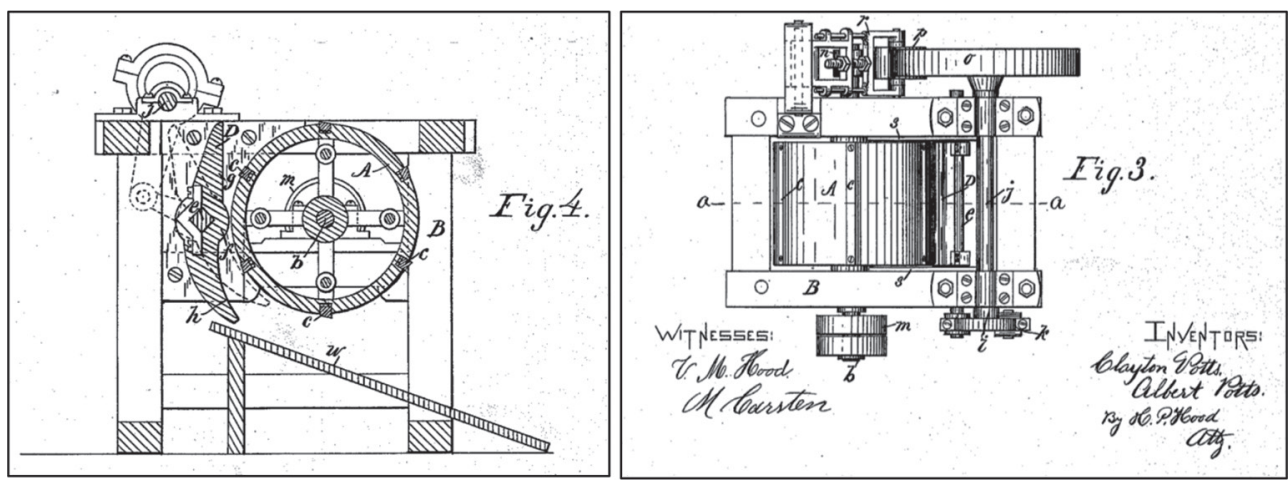

Figure 6. L'invention de Clayton et Albert Potts (Patent $N^{\circ} 322$ 393, 14 juillet 1885)

"This combination consists only of the application of a piece of rubber to one end of the same piece of wood which makes a leadpencil. [...] there might be the advantage of carrying about one instrument instead of two, or of avoiding the liability to loss or misplacing of separate tools. [...] Each, however, continues to perform its own duty, and nothing else. No effect is produced, no result follows, from the joint use of the two $»^{54}$.

Cette fois-ci, le raisonnement ne s'appuie pas uniquement sur la qualification des moyens mis en œuvre, mais stipule une condition particulière sur les effets de l'invention :

"The combination, to be patentable, must produce a different force or effect, or result in the combined forces or processes, from that given by their separate parts. There must be a new result produced by their union: if not so, it is only an aggregation of separate elements ${ }^{55}$.

L'invention doit donc produire un effet supérieur à l'agrégation des éléments individuels ; ce point de raisonnement revient de manière récurrente ${ }^{56}$. Par ailleurs, le jugement précise de nouveau la nature de ces nouveaux effets :

«Perfection of workmanship, however much it may increase the convenience, extend the use, or diminish expense, is not patentable. The distinction between mechanical skill, with its conveniences and advantages, and inventive genius is recognized in all the cases $\gg{ }^{57}$.

Ainsi, non seulement l'invention doit produire un nouvel effet, mais cet effet ne peut être réduit à une amélioration de la technique incluant la facilité d'utilisation, le temps d'utilisation ou le coût lié à la solution technique. L'invention, en tant que mise en œuvre d'éléments déjà connus, doit donc respecter de nouvelles conditions : la combinaison d'éléments, à défaut de créer une modification de l'objet, doit produire une nouvelle fonction synergique au sein du système ; et cet effet doit dépasser une amélioration simple qui est déjà l'effet recherché par l'homme du métier. L'ajout d'une gomme au crayon, ici brevetée, ne peut donc satisfaire cette doctrine : la gomme ne permet pas, en elle-même, d'améliorer la catégorie fonctionnelle des crayons, puisqu'elle produit un effet essentiellement décorrélé de celui du crayon.

La décision Potts v. Creager (1895) adopte de nouveau une évaluation de l'apport fonctionnel mais à partir d'un cas d'application d'une technique connue à une

\footnotetext{
${ }^{54}$ Reckendorfer v. Faber, 92 U.S. 347, 23 L. Ed. 719, 1875 U.S. LEXIS 1765.

55 Ibid.

${ }^{56}$ Hailes v. Von Wormer (1874), Atlantic Works v. Brady (1883), Lincoln Engineering Co. v. Stewart-Warner Co. (1938).

${ }^{57}$ Reckendorfer v. Faber, op. cit.
} 
nouvelle fonction. Cette décision concerne l'invention de Clayton et Albert Potts : une machine visant la désintégration et la pulvérisation d'argile. Ce procédé permet à l'argile d'absorber l'eau plus facilement ce qui rend l'argile plus manipulable. L'invention consiste donc essentiellement en un cylindre tournant à haute vitesse constitué de barres longitudinales sur sa périphérie avec des coins saillants, d'un cylindre fixe situé en parallèle contre lequel l'argile est pressée et enfin d'un système d'approvisionnement de l'argile à l'entrée de la machine (cf. figure 6).

Or Frank F. Creager avait déposé un brevet en 1878 pour une machine dont le principe de fonctionnement était extrêmement similaire (utilisation de barres saillantes en appui contre un support fixe) mais pour le polissage du bois. Le cylindre était mis en rotation et la pression contre la plaque de bois créait une friction permettant le polissage. La Cour suprême doit alors déterminer si effectivement l'invention de C. et A. Potts peut être reconnue nouvelle et valider le brevet étant donné cette antériorité. Après avoir énuméré les antériorités de la technique utilisée, la Cour note tout d'abord qu'aucune de ces inventions de l'état de l'art n'utilise de combinaisons similaires avec pour objectif le travail de l'argile. En l'occurrence, les antériorités ne renvoient pas à une industrie commune, mais sont citées car elles utilisent un principe de cylindre roulant pour différentes applications (broyage de pommes, obtention de pâte de papier, décorticage de graines de coton...). L'état de l'art est donc établi relativement à la fonctionnalité employée dans l'invention et non par rapport à un métier spécifique.

Les différences structurelles entre l'invention de C. et A. Potts et celle de F. Creager sont ensuite explicitées :

"What, then, did the patentees do? They took the cylinder shown in the Creager woodpolishing exhibit, removed the glass bars, and substituted bars of steel; provided it with an abutting surface in the form of a revolving roller, and used it for a totally distinct and different purpose $\gg{ }^{58}$.

Le tribunal reconnaît que les modifications structurelles, en tant que telles, ne sont pas suffisantes pour justifier l'activité inventive, mais les juges remarquent que l'application d'une technique connue dans une branche de l'industrie à une nouvelle application peut justifier d'une activité inventive.

«But where the alleged novelty consists in transferring a device from one branch of industry to another, the answer depends upon a variety of considerations. In such cases we are bound to inquire into the remoteness of relationship of the two industries; what alterations were necessary to adapt the device to its new use, and what the value of such adaptation has been to the new industry ${ }^{59}$.

Les juges mettent donc en avant des conditions liées à l'activité inventive pour les inventions relatives au transfert d'une technique connue vers une nouvelle application. Ces conditions sont qualitatives et renvoient : 1) à l'indépendance ou non entre les deux industries ou applications, 2) aux modifications nécessaires à l'adaptation, 3) à la valeur de l'invention pour la nouvelle fonction visée (en particulier, l'invention présente-t-elle des performances supérieures aux méthodes précédemment utilisées dans l'industrie ?). Dans le cas de l'invention de C. et A. Potts, la «nouveauté » est admise, en particulier car la machine apporte une avancée significative pour le traitement de l'argile. Précisons que ce qui est ici évalué n'est pas la « combinaison » de deux domaines techniques, mais bien un transfert et en particulier l'apport fonctionnel majeur de l'invention pour l'un des deux domaines techniques. Un raisonnement identique est suivi dans Brown v. Piper (1875) ou Atlantic Works v. Brady (1883), concluant cette fois-ci à la non-invention des brevets analysés (cf. tableau 3 ci-dessous).

\footnotetext{
${ }^{58}$ Potts v. Creager, 155 U.S. 597, 15 S. Ct. 194, 39 L. Ed. 275, 1895 U.S. LEXIS 2109.

59 Ibid.
} 


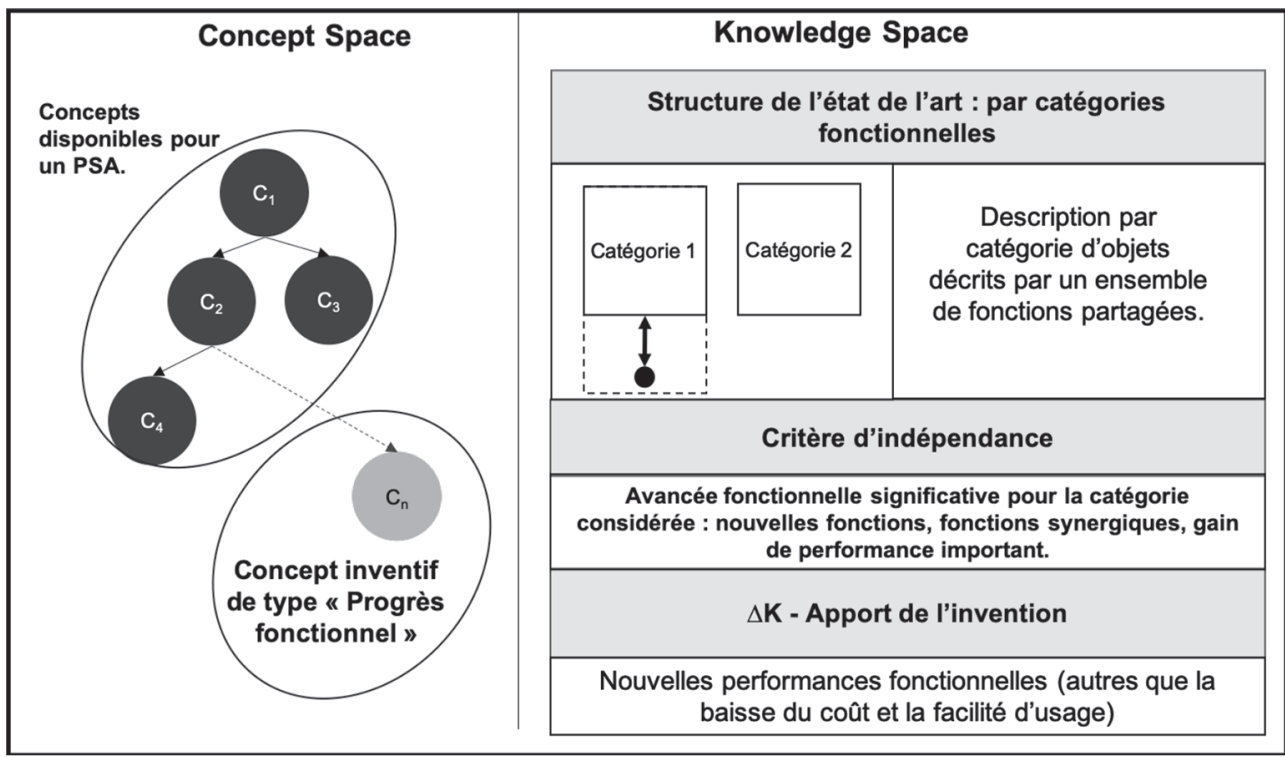

Figure 7. Synthèse du modèle d'invention pour le régime du progrès fonctionnel (1851-1941) interprété $\mathrm{d}$ 'après la théorie $\mathrm{C} / \mathrm{K}$

Ce deuxième régime, que nous nommons régime du progrès fonctionnel, présente donc deux traits particuliers. En premier lieu, la structure de l'état de l'art change : les juges invoquent dans leur raisonnement non plus un objet isolé mais un ensemble d'objets appartenant tous à une même " catégorie fonctionnelle ». Par-là, nous entendons que les juges ne regardent pas chaque objet ou invention de manière individuelle, mais plutôt comme réalisant essentiellement une fonction technique particulière avec un certain niveau de performance, qui définit un état de l'art pertinent. La décision Potts v. Creager est emblématique de ce processus, puisqu'elle cite des objets aux applications diverses mais utilisant un principe fonctionnel unique. En deuxième lieu, le critère d'indépendance s'appuie de fait sur une évaluation « fonctionnelle » des objets : la question n'est plus seulement celle de la nouveauté, ni même de l'utilité, mais bien de la capacité de l'objet à créer un progrès fonctionnel significatif relativement à la catégorie d'objets à laquelle il est rattaché. Plus précisément, les juges cherchent régulièrement à identifier un effet significatif apporté par l'invention qui ne puisse être réduit à l'agrégation d'éléments fonctionnels séparés. Ainsi, ce nouvel effet ne doit pas être indépendant de la catégorie fonctionnelle d'objets à laquelle il s'ajoute ; il doit au contraire venir soutenir, moduler et être subordonné à la fonction « principale » de la catégorie à laquelle le brevet est rattaché (d'où les « fonctions synergiques » qui correspondraient à des fonctions secondaires). Cette recherche est constante et gouverne la majorité des décisions analysées sur cette période (cf. Tableaux 3.1 et 3.2). Ce régime apparaît donc plus complexe que le premier et offre aux juges des tribunaux des États-Unis des critères stables. Cependant, dès 1941, une décision de jurisprudence problématique force de nouveau les juges à réinventer de nouvelles formes d'évaluation. 


\begin{tabular}{|c|c|c|c|c|c|}
\hline $\begin{array}{c}\text { Décisions de } \\
\text { jurisprudence } \\
1851-1941 \\
\text { Régime } \\
\text { du progrès } \\
\text { fonctionnel }\end{array}$ & $\begin{array}{l}\text { Invention } \\
\text { mise en } \\
\text { cause }\end{array}$ & État de l'art & $\begin{array}{l}\text { Structure } \\
\text { d'état de } \\
\text { l'art }\end{array}$ & $\begin{array}{l}\text { Apport de } \\
\text { I'invention }\end{array}$ & $\begin{array}{c}\text { Critère } \\
\text { d'indépen- } \\
\text { dance }\end{array}$ \\
\hline $\begin{array}{l}\text { Hotchkiss v. } \\
\text { Greenwood } \\
\text { (1851) }\end{array}$ & $\begin{array}{l}\text { Poignée en } \\
\text { porcelaine } \\
\text { ou argile avec } \\
\text { système } \\
\text { de fixation }\end{array}$ & $\begin{array}{l}\text { Poignée en } \\
\text { bois ou métal } \\
\text { avec système } \\
\text { de fixation } \\
\text { similaire }\end{array}$ & $\begin{array}{l}\text { Une catégorie } \\
\text { d'objets } \\
\text { (poignées) }\end{array}$ & $\begin{array}{l}\text { Utilisation du } \\
\text { bois ou métal } \\
\text { pour une poi- } \\
\text { gnée avec sys- } \\
\text { tème de fixa- } \\
\text { tion adaptée } \\
\text { pour améliorer } \\
\text { la robustesse et } \\
\text { réduire le coût }\end{array}$ & $\begin{array}{l}\text { Substitution } \\
\text { évidente } \\
\text { car pas de } \\
\text { nouvel effet } \\
\text { hormis le } \\
\text { perfectionne- } \\
\text { ment }\end{array}$ \\
\hline $\begin{array}{l}\text { Stimpson v. } \\
\text { Woodman } \\
\text { (1870) }\end{array}$ & $\begin{array}{l}\text { Machine avec } \\
\text { rouleau métal- } \\
\text { lique à surface } \\
\text { figurée pour le } \\
\text { frottement du } \\
\text { cuir }\end{array}$ & $\begin{array}{l}\text { Rouleau à sur- } \\
\text { face figuré } \\
\text { pour le cuir à } \\
\text { usage manuel } \\
\text { connu et } \\
\text { machine de } \\
\text { traitement du } \\
\text { cuir }\end{array}$ & $\begin{array}{l}\text { Une catégorie } \\
\text { d'objets (outils } \\
\text { de traitement } \\
\text { de cuir) }\end{array}$ & $\begin{array}{l}\text { Combinaison } \\
\text { d'un rouleau } \\
\text { à surface figu- } \\
\text { rée et d'une } \\
\text { machine de } \\
\text { traitement de } \\
\text { cuir pour frot- } \\
\text { ter ce dernier }\end{array}$ & $\begin{array}{l}\text { Substitution } \\
\text { évidente car } \\
\text { la combinai- } \\
\text { son n'apporte } \\
\text { aucun nouvel } \\
\text { effet }\end{array}$ \\
\hline $\begin{array}{l}\text { Rubber-Tip } \\
\text { Pencil } \\
\text { Co. v. Howard } \\
\text { (1874) }\end{array}$ & $\begin{array}{l}\text { Gomme avec } \\
\text { une cavité per- } \\
\text { mettant d'insé- } \\
\text { rer l'extrémité } \\
\text { d'un crayon à } \\
\text { papier retenu } \\
\text { grâce à l'élas- } \\
\text { ticité du caout- } \\
\text { chouc de la } \\
\text { gomme }\end{array}$ & $\begin{array}{l}\text { Les gommes } \\
\text { et leur } \\
\text { propriété } \\
\text { d'élasticité } \\
\text { connues }\end{array}$ & $\begin{array}{l}\text { Une catégorie } \\
\text { d'objets } \\
\text { (gommes de } \\
\text { bureau) }\end{array}$ & $\begin{array}{l}\text { Cavité dans la } \\
\text { gomme utili- } \\
\text { sant l'élasticité } \\
\text { du caoutchouc } \\
\text { pour mainte- } \\
\text { nir un crayon à } \\
\text { papier }\end{array}$ & $\begin{array}{l}\text { Invention évi- } \\
\text { dente car tous } \\
\text { les éléments } \\
\text { sont connus } \\
\text { (gomme, } \\
\text { crayon à } \\
\text { papier, élas- } \\
\text { ticité de la } \\
\text { gomme) et pas } \\
\text { de nouvel effet } \\
\text { obtenu qui ne } \\
\text { soit accessible } \\
\text { à l'homme du } \\
\text { métier }\end{array}$ \\
\hline $\begin{array}{l}\text { Hailes v. Von } \\
\text { Wormer (1874) }\end{array}$ & $\begin{array}{l}\text { Poële à bois dit } \\
\text { "selffeeding } \\
\text { stoves» }\end{array}$ & $\begin{array}{l}\text { Réchaud } \\
\text { (reservoir } \\
\text { stove) et } \\
\text { poële à tirage } \\
\text { réversible }\end{array}$ & $\begin{array}{l}\text { Une catégorie } \\
\text { d'objets } \\
\text { (poëles) }\end{array}$ & $\begin{array}{l}\text { Combinaison } \\
\text { nouvelle des } \\
\text { éléments } \\
\text { contenus dans } \\
\text { le réchaud } \\
\text { et le poële à } \\
\text { tirage réver- } \\
\text { sible permet- } \\
\text { tant d'éviter } \\
\text { une surchauffe } \\
\text { des gaz de } \\
\text { combustion }\end{array}$ & $\begin{array}{l}\text { Invention } \\
\text { nonévidente } \\
\text { car la combi- } \\
\text { naison produit } \\
\text { un nouvel effet }\end{array}$ \\
\hline $\begin{array}{l}\text { Reckendorfer } \\
\text { v. } \\
\text { Faber (1876) }\end{array}$ & $\begin{array}{l}\text { Crayon en bois } \\
\text { disposant à } \\
\text { une extrémité } \\
\text { d'une gomme } \\
\text { insérée dans le } \\
\text { bois du crayon }\end{array}$ & $\begin{array}{l}\text { Ensemble des } \\
\text { gommes et } \\
\text { crayon à bois } \\
\text { connus }\end{array}$ & $\begin{array}{l}\text { Une catégorie } \\
\text { d'objets } \\
\text { (crayons de } \\
\text { bureau) }\end{array}$ & $\begin{array}{l}\text { Combinaison } \\
\text { de la gomme } \\
\text { et de la mine } \\
\text { du crayon } \\
\text { dans le bois du } \\
\text { crayon }\end{array}$ & $\begin{array}{l}\text { Invention évi- } \\
\text { dente car la } \\
\text { combinai- } \\
\text { son ne produit } \\
\text { aucun nouvel } \\
\text { effet }\end{array}$ \\
\hline
\end{tabular}

Tableau 3.1 Description des décisions de jurisprudence pour le régime du progrès fonctionnel (1851 - 1941) 


\begin{tabular}{|c|c|c|c|c|c|}
\hline $\begin{array}{c}\text { Décisions de } \\
\text { jurisprudence } \\
1851 \text { - } 1941 \\
\text { Régime du } \\
\text { progrès } \\
\text { fonctionnel }\end{array}$ & $\begin{array}{l}\text { Invention } \\
\text { mise en } \\
\text { cause }\end{array}$ & État de I'art & $\begin{array}{l}\text { Structure } \\
\text { d'état de } \\
\text { l'art }\end{array}$ & $\begin{array}{l}\text { Apport de } \\
\text { l'invention }\end{array}$ & $\begin{array}{c}\text { Critère } \\
\text { d'indépen- } \\
\text { dance }\end{array}$ \\
\hline $\begin{array}{l}\text { Atlantic Works } \\
\text { v. Brady (1883) }\end{array}$ & $\begin{array}{l}\text { Bateau de } \\
\text { dragage, avec } \\
\text { des réservoirs } \\
\text { d'eau permet- } \\
\text { tant de contrô- } \\
\text { ler la quille et } \\
\text { d'une tige héli- } \\
\text { coïdale relié } \\
\text { aux moteurs } \\
\text { attachés à } \\
\text { l'avant du } \\
\text { bateau permet- } \\
\text { tant de draguer } \\
\text { les fonds }\end{array}$ & $\begin{array}{l}\text { Bateau de } \\
\text { dragage et } \\
\text { sous-systèmes } \\
\text { connus : sys- } \\
\text { tème de } \\
\text { réservoirs } \\
\text { d'eau pour } \\
\text { contrôle de } \\
\text { la quille, tige } \\
\text { hélicoïdale, } \\
\text { machines à } \\
\text { vapeur }\end{array}$ & $\begin{array}{l}\text { Une catégorie } \\
\text { d'objet (bateau } \\
\text { de dragage) et } \\
\text { ses } \\
\text { sous-éléments }\end{array}$ & $\begin{array}{l}\text { Combinaison } \\
\text { d'un bateau } \\
\text { avec sys- } \\
\text { tème de réser- } \\
\text { voirs d'eau et } \\
\text { machines à } \\
\text { vapeurs faisant } \\
\text { tourner une } \\
\text { tige hélicoï- } \\
\text { dale à l'avant } \\
\text { du bateau pour } \\
\text { draguer les } \\
\text { fonds }\end{array}$ & $\begin{array}{l}\text { Invention évi- } \\
\text { dente car la } \\
\text { sélection et la } \\
\text { combinaison } \\
\text { était acces- } \\
\text { sibles à un } \\
\text { homme du } \\
\text { métier }\end{array}$ \\
\hline $\begin{array}{l}\text { Gosnell v. } \\
\text { Bishop (1888) }\end{array}$ & $\begin{array}{l}\text { Tendeur pour } \\
\text { pantalon consti- } \\
\text { tué de pinces } \\
\text { au niveau de } \\
\text { la taille et d'un } \\
\text { système de } \\
\text { tension au } \\
\text { niveau des } \\
\text { genous ser- } \\
\text { vant à tendre le } \\
\text { pantalon }\end{array}$ & $\begin{array}{l}\text { Tendeur pour } \\
\text { pantalon } \\
\text { constitué de } \\
\text { pinces au } \\
\text { niveau de } \\
\text { la taille et } \\
\text { d'une tige file- } \\
\text { tée entre les } \\
\text { pinces servant } \\
\text { à tendre le } \\
\text { pantalon }\end{array}$ & $\begin{array}{l}\text { Une catégorie } \\
\text { d'objets (sys- } \\
\text { tèmes de mise } \\
\text { en tension } \\
\text { pour tendeurs } \\
\text { de pantalon) }\end{array}$ & $\begin{array}{l}\text { Utilisation } \\
\text { du système } \\
\text { pinces et } \\
\text { tiges file- } \\
\text { tée selon un } \\
\text { arrangement } \\
\text { nouveau }\end{array}$ & $\begin{array}{l}\text { Invention non } \\
\text { évidente car le } \\
\text { mécanisme } \\
\text { connu permet } \\
\text { d'obtenir un } \\
\text { effet différent } \\
\text { (tension entre } \\
\text { les genous } \\
\text { et la taille du } \\
\text { pantalon) }\end{array}$ \\
\hline $\begin{array}{l}\text { Potts v. } \\
\text { Creager (1895) }\end{array}$ & $\begin{array}{l}\text { Désintégration } \\
\text { et pulvérisa- } \\
\text { tion de bloc } \\
\text { d'argile à par- } \\
\text { tir de cylindre } \\
\text { coupant, lui } \\
\text { permettant } \\
\text { une absorption } \\
\text { facile de l'eau }\end{array}$ & $\begin{array}{l}\text { - Systèmes } \\
\text { utilisant des } \\
\text { cylindres cou- } \\
\text { pants pour le } \\
\text { polissage du } \\
\text { bois } \\
\text { - Machine } \\
\text { connue de } \\
\text { désintégration } \\
\text { de l'argile }\end{array}$ & $\begin{array}{l}\text { Deux catégo- } \\
\text { ries d'objets } \\
\text { indépendants } \\
\text { (cylindre cou- } \\
\text { pant et outil } \\
\text { de } \\
\text { désintégration } \\
\text { de l'argile) }\end{array}$ & $\begin{array}{l}\text { Emploi du } \\
\text { principe de } \\
\text { cylindre cou- } \\
\text { pant pour un } \\
\text { nouvel usage } \\
\text { (la désintégra- } \\
\text { tion de bloc } \\
\text { d'argile) }\end{array}$ & $\begin{array}{l}\text { Invention } \\
\text { nonévidente } \\
\text { car emploi } \\
\text { d'un prin- } \\
\text { cipe inconnu } \\
\text { (cylindre cou- } \\
\text { pant) pour l'ef- } \\
\text { fet recherché } \\
\text { (désintégration } \\
\text { d'argile) }\end{array}$ \\
\hline $\begin{array}{l}\text { Continental } \\
\text { Paper } \\
\text { Bag Co. v. } \\
\text { Easter } \\
\text { Paper Bag Co. } \\
\text { (1908) }\end{array}$ & $\begin{array}{l}\text { Machine pour } \\
\text { pliage de sacs } \\
\text { en papier com- } \\
\text { binant par une } \\
\text { connexion spéci- } \\
\text { fique un cylindre } \\
\text { rotatif qui met } \\
\text { en mouvement } \\
\text { une plaque de } \\
\text { pliage }\end{array}$ & $\begin{array}{l}\text { Combinaison } \\
\text { de cylindre } \\
\text { rotatif et de } \\
\text { plaque de } \\
\text { pliage par } \\
\text { d'autres } \\
\text { moyens pour } \\
\text { machine de } \\
\text { pliage }\end{array}$ & $\begin{array}{l}\text { Une catégorie } \\
\text { d'objets } \\
\text { (machine } \\
\text { de pliage du } \\
\text { papier) }\end{array}$ & $\begin{array}{l}\text { Modification } \\
\text { du type de } \\
\text { connec- } \\
\text { tion entre le } \\
\text { cylindre rotatif } \\
\text { et la plaque de } \\
\text { pliage }\end{array}$ & $\begin{array}{l}\text { Invention évi- } \\
\text { dente car la } \\
\text { machine de } \\
\text { Continental } \\
\text { Paper Bag est } \\
\text { considérée } \\
\text { identique à } \\
\text { celle de Easter } \\
\text { Paper Bag }\end{array}$ \\
\hline $\begin{array}{l}\text { Lincoln } \\
\text { Engineering } \\
\text { Co v. Stewart- } \\
\text { Warner Corp } \\
\text { (1938) }\end{array}$ & $\begin{array}{l}\text { Pistolet } \\
\text { haute-pression } \\
\text { de lubrification } \\
\text { de roulements } \\
\text { connectés } \\
\text { via un raccord }\end{array}$ & $\begin{array}{l}\text { Dispositif } \\
\text { de pompes } \\
\text { à graisses } \\
\text { incluant les dif- } \\
\text { férents types } \\
\text { de raccords au } \\
\text { roulement }\end{array}$ & $\begin{array}{l}\text { Une catégorie } \\
\text { d'objets } \\
\text { (pompes à } \\
\text { graisses) }\end{array}$ & $\begin{array}{l}\text { Amélioration } \\
\text { du système de } \\
\text { couplage entre } \\
\text { le raccord et le } \\
\text { tuyau de la } \\
\text { pompe }\end{array}$ & $\begin{array}{l}\text { Simple } \\
\text { amélioration } \\
\text { sans apport } \\
\text { d'effet nou- } \\
\text { veau pour un } \\
\text { homme du } \\
\text { métier }\end{array}$ \\
\hline
\end{tabular}

Tableau 3.2 Description des décisions de jurisprudence pour le régime du progrès fonctionnel (1851-1941) 


\section{LE RÉGIME DE L'ORIGINALITÉ COMBINATOIRE (1941- 2007) : LA LÉGALISATION DU CRITÈRE DE "NONOBVIOUSNESS »}

L'introduction du critère de non-évidence aux États-Unis en 1952 est l'aboutissement d'une période de débats vifs autour du critère faisant suite à la décision Cuno Engineering v. Automatic Corp. de 1941, lors de laquelle les juges estiment qu'il est nécessaire pour l'inventeur de démontrer l'existence d'un «flash of creative genius $»^{60}$. Par conséquent, la période de 1941 à 1952 apparaît comme une période de transition jurisprudentielle. En 1950, le juge Learned Hand n'hésite pas à déclarer que le standard juridique de l'invention est devenu « as fugitive, impalpable, wayward, and vague a phantom as exists in the whole paraphernalia of legal concepts $»^{61}$. Le Patent Act de 1952 introduit le critère de nonévidence comme alternative à une évaluation de l'invention jugée impossible. L'homme du métier («person having ordinary skill in the art ») est officiellement défini comme référentiel explicite permettant de juger si l'invention est évidente ou non. Passer du « flash du génie créatif » au concept de nonévidence permet de rationaliser l'évaluation en se focalisant sur un processus plus clair et un ensemble d'indices pouvant montrer que la solution n'était pas triviale ${ }^{62}$.
Une autre modification institutionnelle majeure intervient quelques décennies plus tard. En 1982, les États-Unis mettent en place une cour d'appel centralisée pour les tribunaux fédéraux (Court of Appeals for the Federal Circuit) dont l'influence sur le système des brevets est majeure ${ }^{63}$ : cette cour adopte une approche « pro-brevet » en rupture avec les pratiques des cours précédentes. Cette « cour amicale » interprète plus largement les droits conférés par le brevet ${ }^{64}$, elle est plus encline à accepter la mise en place d'injonctions préliminaires (interdiction temporaire d'exploitation d'une invention prononcée par la cour en cas de soupçon important de contrefaçon), et offre des dommages et intérêts plus importants aux détenteurs de droits en cas de violation effective d'un brevet. Ces modifications s'accompagnent d'une augmentation spectaculaire du nombre de demandes de brevets aux États-Unis : en 1963, 85869 demandes de brevets sont déposées à l'USPTO, en 2015 l'office reçoit 589410 demandes (uniquement pour les « utility patents »). On assiste simultanément à une augmentation très considérable du nombre d'examinateurs de brevets à l'USPTO et à la stabilisation relative d'une classification des brevets modernes appelée USPC (United States Patent Classification). Se produit donc une « industrialisation » rapide de l'évaluation des brevets, qui conduit à la standardisation des procédures, comme le prouve la publication chaque année depuis 1948 des Manuals of Patent Examining Procedure (MPEP) ${ }^{65}$. De

\footnotetext{
${ }^{60}$ Cuno Engineering v. Automatic Corp, 314 U.S. 84, 62 S. Ct. 37, 86 L. Ed. 58, 1941 U.S. LEXIS 1250.

${ }^{61}$ Décision du juge Learned Hand dans Harries v. Air King Prods. Co., 183 F.2d 158, 162 (2d Cir. 1950), citée dans A. L. Landers, " Ordinary Creativity in Patent Law: The Artist Within the Scientist », art. cit.

${ }^{62}$ Pour la description de ce processus et des indices pouvant être mobilisés par les examinateurs de brevets américains, voir « Manual of Patent Examining Procedure », $9^{\text {th }}$ Edition (revised in January 2018), Chapitre 2100 « Patentability », Section $2141-2144$.

${ }^{63}$ S. Kortum et J. Lerner, « What is behind the Recent Surge in Patenting? », Research Policy, vol. 28, n 1, 1999, p. 1-22 ; B. H. Hall, « Exploring the Patent Explosion », The Journal of Technology Transfer, vol. 30, ${ }^{\circ} 1-2,2004$, p. $35-48$.

${ }^{64}$ B. H. Hall et R. H. Ziedonis, « The Patent Paradox Revisited: an Empirical Study of Patenting in the US Semiconductor Industry, 1979-1995 », RAND Journal of Economics, vol. 32, n 1, 2001, p. 101-128.

${ }^{65}$ Dès 1948 , le MPEP introduit d'ailleurs le critère « d'invention » au côté du critère de nouveauté et d'utilité. Voir Manual of Patent Examining Procedure, 1948, Section 3, "Patentable Subject Matter », §11 Invention : «It is not enough that a thing shall be new, in the sense that in the shape of form which it is produced, it shall not have been
} 
fait, dès 1966 les tribunaux rationalisent aussi l'évaluation du critère de non-évidence. Pour présenter les spécificités du modèle d'invention de cette période, nous présenterons en détail trois décisions majeures : Cuno Engineering Corp. v. Automatic Corp. (1941), Graham v. John Deere (1966) et ACS Hospital System v. Montefiore Hospital and Wells National Services Corp. $(1984)^{66}$.

La décision Cuno Engineering Corp. v. Automatic Corp. traite d'une invention portant sur un allume-cigare électrique pour le secteur automobile. Ce dernier a la particularité d'être couplé à un contrôleur thermostatique permettant à l'utilisateur d'activer l'allumecigare, et d'attendre simplement que ce dernier atteigne la température adéquate sans avoir à éteindre le système par lui-même.

1) État de l'art : Les juges se réfèrent à plusieurs allume-cigares antérieurs utilisant des systèmes de bouton-poussoir que l'automobiliste devait activer et désactiver :

"Several types of the "wireless" or "cordless" lighters appeared. [...] In Zecchini (No. $1,437,701)$ the operator pressed and held down a push-button to close the circuit. In Metzger (No. 1,622,334) the operator closed the circuit by depressing and rotating the plug ${ }^{67}$.

D'autres antériorités, relatives non à l'allume-cigare mais au contrôleur thermostatique, sont également citées :

« Harley, in 1907 (No. 852,326), included such a thermostat in an electric heater for vulcanizing, so as to limit automatically the temperature attainable. Andrews, in 1912 (No. 1,025,852), showed a bimetallic thermostat in an electrical flat iron, designed to open the circuit at a predetermined temperature. In 1919, Newsom (No. 1,318,168), showed an electric coffee cooker in which a thermostat, actuated by the temperature within the receptacle, operated to open and close the circuit intermittently ${ }^{68}$.

L'état de l'art ne se réduit donc plus à une seule catégorie d'inventions associées au brevet examiné, mais s'étend aux combinaisons déjà existantes du contrôleur thermostatique avec des objets éloignés du secteur automobile. Par ailleurs, ces différents éléments de l'état de l'art sont considérés explicitement comme des éléments de connaissances d'un homme du métier et l'invention est évaluée à partir de ceux-ci.

2) Apport de l'invention : le tribunal énonce ainsi la nouveauté du brevet, objet du litige :

"Mead added to the so-called "wireless" or "cordless" lighter a thermostatic control responsive to the temperature of the heating coil ${ }^{69}$.

La nouveauté est donc ici exprimée en termes de combinaisons d'éléments techniques (et non en termes de performance, ou de modification de propriétés propre à l'allume-cigare).

3) Critère d'indépendance : le brevet déposé par Copeland combine déjà un allume-cigare avec un contrôleur thermostatique, ce qui poussera la cour à trancher ainsi la question de l'indépendance :

«As we have shown, both the thermostatically controlled heating unit and the lighter with a removable plug bearing the heating unit were disclosed by the prior art. More must be done

\footnotetext{
before known, and that it shall be useful, but it must, under the Constitution and the statute, amount to an invention or discovery $\gg$.

${ }^{66}$ Nous n'incluons pas dans notre analyse l'émergence de la doctrine des équivalences à partir des années 1950 aux États-Unis pour des raisons de synthèse ; on peut indiquer que le raisonnement appliqué par les juges pour l'analyse de l'équivalence présente de nombreux points communs avec celui de la non-évidence, cependant la doctrine de l'équivalence s'applique à évaluer la portée d'un brevet vis-à-vis d'une contrefaçon. Voir J. R. Allison et M. A. Lemley, «The (Unnoticed) Demise of the Doctrine of Equivalents », Stanford Law Review, vol. 59, n 4, 2007, p. 955-984.

${ }^{67}$ Cuno Engineering v. Automatic Corp., op. cit.

${ }^{68}$ Ibid.

${ }^{69}$ Ibid.
} 
than to utilize the skill of the art in bringing old tools into new combinations ${ }^{70}$.

L'évaluation de l'invention fait donc explicitement l'objet d'une nouvelle question qui deviendra l'équivalent moderne du critère d'activité inventive, distinct de la nouveauté et de l'utilité :

«We may concede that the functions performed by Mead's combination were new and useful. But that does not necessarily make the device patentable. Under the statute (35 U.S.C. \$ 31; R.S. § 4886) the device must not only be "new and useful", it must also be an "invention" or "discovery". [...] That is to say, the new device, however useful it may be, must reveal the flash of creative genius $\gg{ }^{71}$.

Cette décision se traduit donc par quatre changements majeurs : 1) l'état de l'art mobilisé ne se réduit plus seulement à une catégorie d'inventions proches, mais s'étend à des catégories d'inventions combinant des éléments similaires (ici le contrôleur thermostatique) et pouvant être très hétérogènes (radiateurs, cafetières, etc.) ; 2) l'apport de l'invention est explicitement décrit comme la combinaison de deux éléments techniques ; 3) l'évaluation de la nouveauté ne suffit plus, une nouvelle évaluation est effectuée à part entière : celle de l'inventivité de la solution; 4) l'homme du métier est capable de combiner des éléments de l'état de l'art, si ceux-ci ont déjà été combinés, indépendamment de l'avancée fonctionnelle que l'invention peut produire. Ces changements peuvent être repérés dans un ensemble de jugements prononcés entre 1941 et 2007 (cf. tableaux 4.1 et 4.2).

L'introduction du « critère d'invention » associée au terme ambigu et difficilement interprétable de "flash of creative genius » entraîne des débats intenses au sein des tribunaux ${ }^{72}$. En 1949, lors de la décision Jungersen v. Ostby, le juge Jackson s'oppose à la décision rendue par la Cour suprême, en pointant du doigt la «passion » des tribunaux pour l'annulation des brevets ${ }^{73}$. En 1952, en réponse à cette incertitude juridique, est introduit dans la loi un critère dit de « nonévidence », éliminant le terme d'invention jugé trop polémique. La première interprétation jurisprudentielle de ce nouveau standard juridique a lieu lors du procès Graham v. John Deere (1966).

Dans ce cas, la décision rendue par la Cour suprême répond dans le même jugement à plusieurs procès : Graham v. John Deere, Calmar Inc. v. Cook Chemical Co., ColgatePalmolive Co. v. Cook Chemical Co. Puisque les raisonnements appliqués sont extrêmement similaires, nous ne décrirons que le premier. Avant d'établir le jugement, la Cour suprême interprète la loi et en déduit une évaluation de l'évidence divisée en plusieurs étapes. Cette méthode est aujourd'hui le schéma moderne d'évaluation du critère de " nonobviousness » aux États-Unis. La méthode est la suivante : 1) l'étendue et le contenu de l'état de l'art doivent être identifiés ; 2) les différences entre l'état de l'art identifié et les revendications doivent être explicitées ; 3) le niveau de compétence de l'homme du métier doit être analysé. À partir de ces étapes, l'évidence ou non du brevet est déterminée. Bien que la structure générale soit similaire à celle généralement adoptée par les tribunaux lors de l'évaluation, on a là une standardisation de la procédure censée éviter des raccourcis lors de l'évaluation ou de la prise en compte de facteurs non-pertinents. Par ailleurs, cette évaluation peut alors être guidée par des considérations secondaires, appelées Graham Factors : succès commercial, identification d'un besoin encore non satisfait,

\footnotetext{
${ }^{70}$ Ibid.

${ }^{71}$ Ibid.

72 J. F. Duffy, « Inventing Invention: a Case Study of Legal Innovation », art. cit.

${ }^{73}$ Jungersen v. Ostby \& Barton Co. 335 U.S. 560, 69 S. Ct. 269, 93 L. Ed. 2d 235, 1949 U.S. LEXIS 3052. Le juge Jackson contestant la décision : «But I doubt that the remedy for such Patent Office passion for granting patents is an equally strong passion in this Court for striking them down so that the only patent that is valid is one which this Court has not been able to get its hands on ».
} 
échecs d'autres concepteurs à fournir une solution. Ces facteurs offrent donc des indices à l'examinateur concernant l'inventivité de la solution contenue dans le brevet.

\begin{tabular}{|c|c|c|c|c|c|}
\hline $\begin{array}{c}\text { Décisions de } \\
\text { jurisprudence } \\
1941 \text { - } 2007 \\
\text { Régime de } \\
\text { l'originalité } \\
\text { combinatoire }\end{array}$ & $\begin{array}{l}\text { Invention } \\
\text { mise en } \\
\text { cause }\end{array}$ & État de I'art & $\begin{array}{l}\text { Structure } \\
\text { d'état de } \\
\text { l'art }\end{array}$ & $\begin{array}{l}\text { Apport de } \\
\text { l'invention }\end{array}$ & $\begin{array}{c}\text { Critère } \\
\text { d'indépen- } \\
\text { dance }\end{array}$ \\
\hline $\begin{array}{l}\text { Cuno } \\
\text { Engineering } \\
\text { v. Automatic } \\
\text { Corp. } \\
\text { (1941) }\end{array}$ & $\begin{array}{l}\text { Allume-cigare } \\
\text { électrique avec } \\
\text { contrôleur } \\
\text { thermostatique }\end{array}$ & $\begin{array}{l}\text { - Allume-cigare } \\
\text { électrique à } \\
\text { boutonpoussoir } \\
\text { - Contrôleur } \\
\text { thermostatique } \\
\text { - Divers objets } \\
\text { combinés } \\
\text { (grille-pain, } \\
\text { radiateur } \\
\text { électrique...) }\end{array}$ & $\begin{array}{l}\text { Trois catégo- } \\
\text { ries d'inven- } \\
\text { tions avec des } \\
\text { inventions les } \\
\text { combinant }\end{array}$ & $\begin{array}{l}\text { Combinaison } \\
\text { de l'allume- } \\
\text { cigare avec un } \\
\text { contrôleur } \\
\text { thermostatique }\end{array}$ & $\begin{array}{l}\text { Invention évi- } \\
\text { dente car } \\
\text { I'utilisation } \\
\text { du contrô- } \\
\text { leur thermos- } \\
\text { tatique en } \\
\text { combinaison } \\
\text { de système } \\
\text { chauffant était } \\
\text { connue }\end{array}$ \\
\hline $\begin{array}{l}\text { Jungersen } \\
\text { v. Ostby } \\
\text { (1949) }\end{array}$ & $\begin{array}{l}\text { Méthode pour } \\
\text { la réalisation } \\
\text { d'un moule de } \\
\text { grande préci- } \\
\text { sion pour la } \\
\text { joaillerie }\end{array}$ & $\begin{array}{l}\text { - Réalisation } \\
\text { de moules } \\
\text { pour le métal: } \\
\text { utilisation de } \\
\text { la force centri- } \\
\text { fuge pour } \\
\text { introduire } \\
\text { le métal en } \\
\text { fusion dans le } \\
\text { contremoule } \\
\text { - Réalisation } \\
\text { de moule pour } \\
\text { la joaillerie: } \\
\text { méthode de la } \\
\text { cire perdue }\end{array}$ & $\begin{array}{l}\text { Une catégorie } \\
\text { d'invention } \\
\text { séparée en } \\
\text { deux sous- } \\
\text { catégories } \\
\text { combinées } \\
\text { (moules pour } \\
\text { le métal et } \\
\text { moules pour la } \\
\text { joaillerie) }\end{array}$ & $\begin{array}{l}\text { Combinaison } \\
\text { de la méthode } \\
\text { de la cire per- } \\
\text { due et utili- } \\
\text { sation de la } \\
\text { force centri- } \\
\text { fuge pour pla- } \\
\text { quer la cire } \\
\text { fondue dans le } \\
\text { contre-moule }\end{array}$ & $\begin{array}{l}\text { Invention évi- } \\
\text { dente car la } \\
\text { combinaison } \\
\text { entre les tech- } \\
\text { niques liées } \\
\text { aux moules } \\
\text { de métaux } \\
\text { et moules de } \\
\text { joaillerie évi- } \\
\text { dente car ils } \\
\text { consti- } \\
\text { tuent des } \\
\text { connaissances } \\
\text { communes à } \\
\text { l'art du mou- } \\
\text { lage pour un } \\
\text { homme du } \\
\text { métier }\end{array}$ \\
\hline $\begin{array}{l}\text { A \& P Co. Tea } \\
\text { v. Supermarket } \\
\text { Corp. } \\
\text { (1951) }\end{array}$ & $\begin{array}{l}\text { Comptoir de } \\
\text { caisse avec un } \\
\text { tiroir coulis- } \\
\text { sant permet- } \\
\text { tant au caissier } \\
\text { de ramener } \\
\text { les achats du } \\
\text { consomma- } \\
\text { teur à son } \\
\text { niveau }\end{array}$ & $\begin{array}{l}\text { Comptoir de } \\
\text { caisse connu } \\
+ \text { système de } \\
\text { tiroir coulis- } \\
\text { sant connu }\end{array}$ & $\begin{array}{l}\text { Deux catégo- } \\
\text { ries d'inven- } \\
\text { tions (comptoir } \\
\text { de caisse et } \\
\text { système de } \\
\text { tiroirs) }\end{array}$ & $\begin{array}{l}\text { Elongation du } \\
\text { comptoir et } \\
\text { combinaison } \\
\text { de ce comptoir } \\
\text { avec le sys- } \\
\text { tème de tiroir } \\
\text { coulissant }\end{array}$ & $\begin{array}{l}\text { Invention évi- } \\
\text { dente car la } \\
\text { combinaison } \\
\text { n'apporte } \\
\text { aucun nou- } \\
\text { vel effet spé- } \\
\text { cifique à la } \\
\text { combinai- } \\
\text { son des deux } \\
\text { techniques }\end{array}$ \\
\hline $\begin{array}{l}\text { Graham v. } \\
\text { John Deere } \\
(1966)\end{array}$ & $\begin{array}{l}\text { Système de } \\
\text { charnière à } \\
\text { ressort pour } \\
\text { bras de labour }\end{array}$ & $\begin{array}{l}\text { Charnière à } \\
\text { ressort avec } \\
\text { fixation du } \\
\text { bras au-dessus } \\
\text { du pivot de la } \\
\text { charnière }\end{array}$ & $\begin{array}{l}\text { Une catégorie } \\
\text { d'inventions } \\
\text { (charnière à } \\
\text { ressort) }\end{array}$ & $\begin{array}{l}\text { Réduction de } \\
\text { I'usure par } \\
\text { modification } \\
\text { de la posi- } \\
\text { tion du bras } \\
\text { par rapport } \\
\text { au pivot de la } \\
\text { charnière }\end{array}$ & $\begin{array}{l}\text { Invention évi- } \\
\text { dente car un } \\
\text { homme du } \\
\text { métier pou- } \\
\text { vait modifier la } \\
\text { position avec } \\
\text { pour objec- } \\
\text { tif de réduire } \\
\text { l'usure }\end{array}$ \\
\hline
\end{tabular}

Tableau 4.1 Description des décisions de jurisprudence pour le régime de I'originalité combinatoire (1941-2007) 


\begin{tabular}{|c|c|c|c|c|c|}
\hline $\begin{array}{c}\text { Décisions de } \\
\text { jurisprudence } \\
1941 \text { - } 2007 \\
\text { Régime de } \\
\text { l'originalité } \\
\text { combinatoire }\end{array}$ & $\begin{array}{l}\text { Invention } \\
\text { mise en } \\
\text { cause }\end{array}$ & État de l'art & $\begin{array}{l}\text { Structure } \\
\text { d'état de } \\
\text { I'art }\end{array}$ & $\begin{array}{l}\text { Apport de } \\
\text { l'invention }\end{array}$ & $\begin{array}{l}\text { Critère } \\
\text { d'indépen- } \\
\text { dance }\end{array}$ \\
\hline $\begin{array}{l}\text { United States } \\
\text { v. Adams } \\
\text { (1966) }\end{array}$ & $\begin{array}{l}\text { Batterie élec- } \\
\text { trique non- } \\
\text { rechargeable } \\
\text { constituée } \\
\text { d'une élec- } \\
\text { trode de } \\
\text { magnésium } \\
\text { et d'une élec- } \\
\text { trode de chlo- } \\
\text { rure de cuivre } \\
\text { activable par } \\
\text { ajout d'eau } \\
\text { (ou eau salée) } \\
\text { en guise } \\
\text { d'électrolyte }\end{array}$ & $\begin{array}{l}\text { Différents } \\
\text { types de bat- } \\
\text { teries acti- } \\
\text { vées par élec- } \\
\text { trolyte à base } \\
\text { d'acides avec } \\
\text { les types } \\
\text { d'électro- } \\
\text { des associées } \\
\text { (zinc, chlorure } \\
\text { d'argent, } \\
\text { magnesium) }\end{array}$ & $\begin{array}{l}\text { Une catégorie } \\
\text { d'inventions } \\
\text { séparée en } \\
\text { deux sous- } \\
\text { catégories } \\
\text { (batteries à } \\
\text { eau et batte- } \\
\text { ries à acide) }\end{array}$ & $\begin{array}{l}\text { Batterie acti- } \\
\text { vée à l'eau } \\
\text { délivrant } \\
\text { une tension } \\
\text { constante } \\
\text { fonction- } \\
\text { nant dans } \\
\text { une grande } \\
\text { plage de } \\
\text { températures }\end{array}$ & $\begin{array}{l}\text { Invention } \\
\text { reconnue non } \\
\text { évidente car } \\
\text { la combinai- } \\
\text { son d'élec- } \\
\text { trodes adé- } \\
\text { quates avec } \\
\text { un système } \\
\text { d'électrolyte } \\
\text { à eau n'était } \\
\text { pas connue } \\
\text { par l'homme } \\
\text { du métier } \\
\text { (voire les } \\
\text { enseignements } \\
\text { empêchaient } \\
\text { une telle } \\
\text { combinaison). }\end{array}$ \\
\hline $\begin{array}{l}\text { Anderson's } \\
\text { Black Rock } \\
\text { v. Pavement } \\
\text { Salvage (1969) }\end{array}$ & $\begin{array}{l}\text { Combinaison } \\
\text { d'un brû- } \\
\text { leur à chaleur } \\
\text { radiante et } \\
\text { d'une machine } \\
\text { de dépôt et } \\
\text { mise en forme } \\
\text { d'asphalte }\end{array}$ & $\begin{array}{l}\text { Brûleur à cha- } \\
\text { leur radiante } \\
\text { connu (y com- } \\
\text { pris pour le } \\
\text { chauffage } \\
\text { ponctuel de } \\
\text { l'asphalte) } \\
\text { - Machine de } \\
\text { dépôt d'as- } \\
\text { phalte avec } \\
\text { dameuse et } \\
\text { chape pour la } \\
\text { mise en forme }\end{array}$ & $\begin{array}{l}\text { Deux caté- } \\
\text { gories d'in- } \\
\text { ventions } \\
\text { déjà utilisées } \\
\text { conjointement }\end{array}$ & $\begin{array}{l}\text { Fixation du } \\
\text { brûleur à cha- } \\
\text { leur radiante } \\
\text { sur le côté de } \\
\text { la machine de } \\
\text { dépôt pour } \\
\text { continûment } \\
\text { chauffer l'as- } \\
\text { phalte et le } \\
\text { déposer (meil- } \\
\text { leur joint entre } \\
\text { les couches } \\
\text { d'asphalte) }\end{array}$ & $\begin{array}{l}\text { Invention évi- } \\
\text { dente car la } \\
\text { combinaison } \\
\text { des deux fonc- } \\
\text { tions (dépôt } \\
\text { d'asphalte } \\
\text { et chauffage) } \\
\text { étaient déjà } \\
\text { connue }\end{array}$ \\
\hline $\begin{array}{l}\text { Sakraida } \\
\text { v. Ag Pro } \\
\text { (1976) }\end{array}$ & $\begin{array}{l}\text { Système de } \\
\text { rinçage à l'eau } \\
\text { pour le sol } \\
\text { d'une étable } \\
\text { laitière consti- } \\
\text { tué d'un } \\
\text { réservoir d'eau } \\
\text { ouvert abrup- } \\
\text { tement par } \\
\text { une trappe, } \\
\text { permettant } \\
\text { un nettoyage } \\
\text { rapide }\end{array}$ & $\begin{array}{l}\text { - Réservoirs } \\
\text { d'eau } \\
\text { connus et } \\
\text { leurs } \\
\text { systèmes } \\
\text { d'ouverture } \\
\text { - Système de } \\
\text { rinçage }\end{array}$ & $\begin{array}{l}\text { Deux } \\
\text { catégories } \\
\text { d'inventions } \\
\text { déjà } \\
\text { combinées }\end{array}$ & $\begin{array}{l}\text { Obtention } \\
\text { d'un nettoyage } \\
\text { rapide grâce } \\
\text { à un lâcher } \\
\text { d'eau mas- } \\
\text { sif directe- } \\
\text { ment depuis le } \\
\text { réservoir situé } \\
\text { en hauteur }\end{array}$ & $\begin{array}{l}\text { Invention évi- } \\
\text { dente car la } \\
\text { combinaison } \\
\text { (réservoirs } \\
\text { et systèmes } \\
\text { d'ouverture) } \\
\text { était déjà } \\
\text { connue et ici } \\
\text { aucun effet } \\
\text { technique } \\
\text { nouveau n'est } \\
\text { créé (création } \\
\text { d'un fort débit } \\
\text { d'eau) }\end{array}$ \\
\hline
\end{tabular}




\begin{tabular}{|c|c|c|c|c|c|}
\hline $\begin{array}{c}\text { Décisions de } \\
\text { jurisprudence } \\
1941 \text { - } 2007 \\
\text { Régime de } \\
\text { l'originalité } \\
\text { combinatoire }\end{array}$ & $\begin{array}{l}\text { Invention } \\
\text { mise en } \\
\text { cause }\end{array}$ & État de l'art & $\begin{array}{c}\text { Structure } \\
\text { d'état de } \\
\text { l'art }\end{array}$ & $\begin{array}{l}\text { Apport de } \\
\text { I'invention }\end{array}$ & $\begin{array}{l}\text { Critère } \\
\text { d'indépen- } \\
\text { dance }\end{array}$ \\
\hline $\begin{array}{l}\text { ACS Hosp. } \\
\text { System } \\
\text { v. Montefiore } \\
\text { Hosp. and } \\
\text { Wells } \\
\text { N.S. Corp. } \\
\text { (1984) }\end{array}$ & $\begin{array}{l}\text { Système de } \\
\text { location de } \\
\text { télévision } \\
\text { avec interrup- } \\
\text { teur prioritaire } \\
\text { actionnable } \\
\text { par l'utilisateur }\end{array}$ & $\begin{array}{l}\text { - Système } \\
\text { de location } \\
\text { de télévision } \\
\text { connu } \\
\text { - Système } \\
\text { d'interrupteur } \\
\text { prioritaire } \\
\text { connu }\end{array}$ & $\begin{array}{l}\text { Deux } \\
\text { catégories } \\
\text { d'inventions } \\
\text { indépendantes }\end{array}$ & $\begin{array}{l}\text { Combinaison } \\
\text { du système } \\
\text { de location de } \\
\text { télévision avec } \\
\text { l'interrupteur } \\
\text { permettant à } \\
\text { un utilisateur } \\
\text { d'activer le } \\
\text { service sans } \\
\text { attendre l'in- } \\
\text { tervention } \\
\text { d'un opérateur }\end{array}$ & $\begin{array}{l}\text { Invention } \\
\text { non évidente } \\
\text { car aucune } \\
\text { connaissance } \\
\text { de l'état de } \\
\text { l'art ne permet } \\
\text { d'identifier la } \\
\text { combinaison } \\
\text { comme déjà } \\
\text { connue }\end{array}$ \\
\hline $\begin{array}{l}\text { In Re Kahn } \\
\text { (2006) }\end{array}$ & $\begin{array}{l}\text { Système de } \\
\text { lecture de } \\
\text { texte par syn- } \\
\text { thèse vocale } \\
\text { pour personne } \\
\text { aveugle avec } \\
\text { contrôle de la } \\
\text { zone à lire par } \\
\text { "eye-tracking", } \\
\text { et génération } \\
\text { de sons "loca- } \\
\text { lisés" permet- } \\
\text { tant de déter- } \\
\text { miner la zone } \\
\text { lue }\end{array}$ & $\begin{array}{l}\text { - Système } \\
\text { de sélec- } \\
\text { tion de zone } \\
\text { de l'écran par } \\
\text { "eye-tracking" } \\
\text { connu } \\
\text { - Système de } \\
\text { lecture par } \\
\text { synthèse vocal } \\
\text { connu } \\
\text { - Système de } \\
\text { génération de } \\
\text { sons localisés }\end{array}$ & $\begin{array}{l}\text { Trois catégo- } \\
\text { ries d'inven- } \\
\text { tions, avec des } \\
\text { éléments } \\
\text { suggérant leur } \\
\text { combinaison }\end{array}$ & $\begin{array}{l}\text { Combinaison } \\
\text { des différents } \\
\text { systèmes exis- } \\
\text { tants pour l'ap- } \\
\text { plication aux } \\
\text { personnes } \\
\text { aveugles leur } \\
\text { donnant une } \\
\text { sensation de } \\
\text { "lecture» } \\
\text { par le regard }\end{array}$ & $\begin{array}{l}\text { Invention évi- } \\
\text { dente car la } \\
\text { combinaison } \\
\text { avait déjà été } \\
\text { suggérée à } \\
\text { I'homme du } \\
\text { métier par les } \\
\text { concepteurs } \\
\text { des différents } \\
\text { systèmes }\end{array}$ \\
\hline
\end{tabular}

Tableau 4.2 Description des décisions de jurisprudence pour le régime de l'originalité combinatoire (1941-2007)

Après avoir établi cette méthode d'évaluation, la Cour suprême revient sur l'invention de William T. Graham. Celle-ci porte sur l'amélioration (réduction de l'usure) d'un système de charnières à ressort pour un bras de labour permettant à ce dernier d'amortir les chocs lors de son utilisation sur des sols rocailleux. L'apport du brevet de Graham porte sur le placement de la tige qui pivote lorsque le bras rattaché au système rencontre des roches (cf. figure 8). Cette tige est située sous le système de charnière, rattaché par un support, ce qui lui permet de pivoter librement et lui assure une libre flexion en cas d'effort soutenu. Nous ne revenons pas sur la description de l'état de l'art, mais étudions le discours tenu par les juges pour établir la non-évidence. Concernant ce dernier, la cour développe le raisonnement suivant :

"If free-flexing, as petitioners now argue, is the crucial difference above the prior art, then it appears evident that the desired result would be obtainable by not boxing the shank within the confines of the hinge. [...] Certainly, a person having ordinary skill in the prior art, given the fact that the flex in the shank could be utilized more effectively if allowed to run the entire length of the shank, would immediately see that the thing to do was what Graham did, i.e., invert the shank and the hinge plate. ")

L'homme du métier est donc capable, à partir des connaissances disponibles, de 


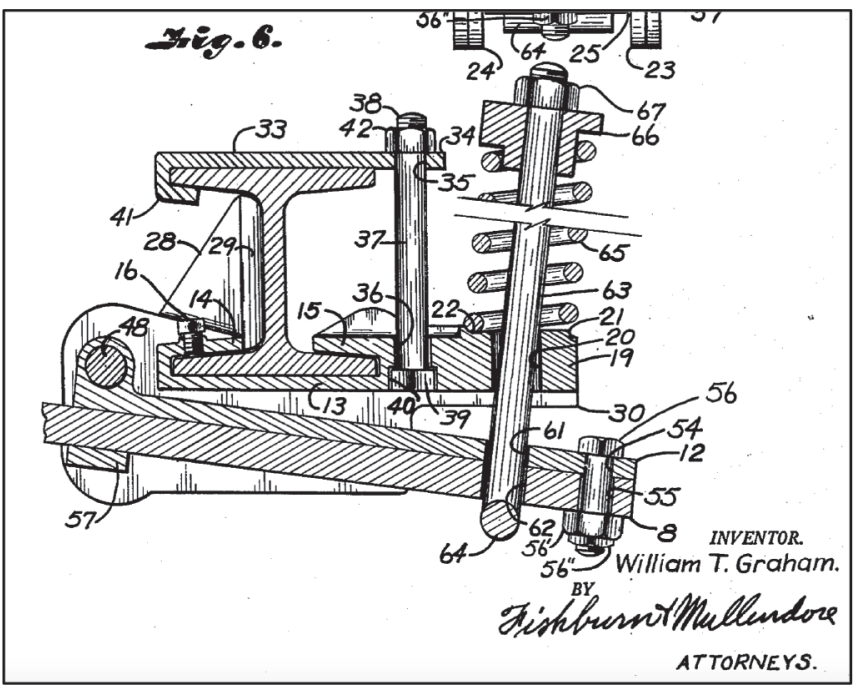

Figure 8. Vue de côté, brevet de Graham

résoudre certains problèmes si les éléments de la solution lui sont connus, et que ces éléments ont déjà été combinés. L'état de l'art et les combinaisons connues constituent donc une base de connaissances pour l'homme du métier à partir de laquelle il peut modifier des éléments techniques du système pour l'obtention d'effets connus. En l'occurrence, l'opération effectuée (" invert the shank and the hinge plate ») est considérée comme atteignable par l'homme du métier, simplement en admettant qu'il était évident que si les éléments étaient connus et le problème clairement formulé, cette opération apparaissait en un sens triviale. À partir des années 1960, la détermination de l'évidence reste relativement peu décrite. Différentes décisions suggèrent que les raisonnements qui ont précédé 1952 restent similaires (Anderson's Black Rock v. Pavement Salvage (1969), Sakraida v. Ag Pro (1976)) (cf. tableaux 4.1 et 4.2). Cependant une différence majeure intervient : les juges justifient souvent l'évidence de combinaison par le fait que certains enseignements de l'état de l'art auraient poussé l'homme du métier à obtenir l'invention revendiquée ${ }^{74}$.

En 1984, ce raisonnement se formalise et prend la forme du test Teaching-SuggestionMotivation (TSM) mis en place non par la Cour suprême mais par les cours fédérales dans la décision ACS Hospital System v. Montefiore Hospital and Wells National Services Corp. (Federal Circuit, 1984). Le brevet déposé par ACS porte sur un système de location de télévision comprenant un interrupteur actionnable par l'opérateur, un interrupteur prioritaire actionnable par l'utilisateur et un signal lumineux indiquant l'activation de l'interrupteur prioritaire. L'invention permet à un utilisateur de louer la télévision sans avoir à attendre une activation à distance de la télévision. Le jugement indique que les

\footnotetext{
${ }^{74}$ Anderson's-Black Rock, Inc. v. Pavement Salvage Co., 396 U.S. 57, 90 S. Ct. 305, 24 L. Ed. 2d 258, 1969 U.S. LEXIS 3322. Le juge Douglas énonçant le jugement : «We conclude that while the combination of old elements performed a useful function, it added nothing to the nature and quality of the radiant-heat burner already patented $»$; Sakraida v. Ag Pro, Inc., 425 U.S. 273, 96 S. Ct. 1532, 47 L. Ed. 2d 784, 1976 U.S. LEXIS 146 : le juge Brennan énonçant le jugement : " this patent simply arranges old elements with each performing the same function it had been known to perform, although perhaps producing a more striking result than in previous combinations. Such combinations are not patentable under standards appropriate for a combination patent $»$.
} 


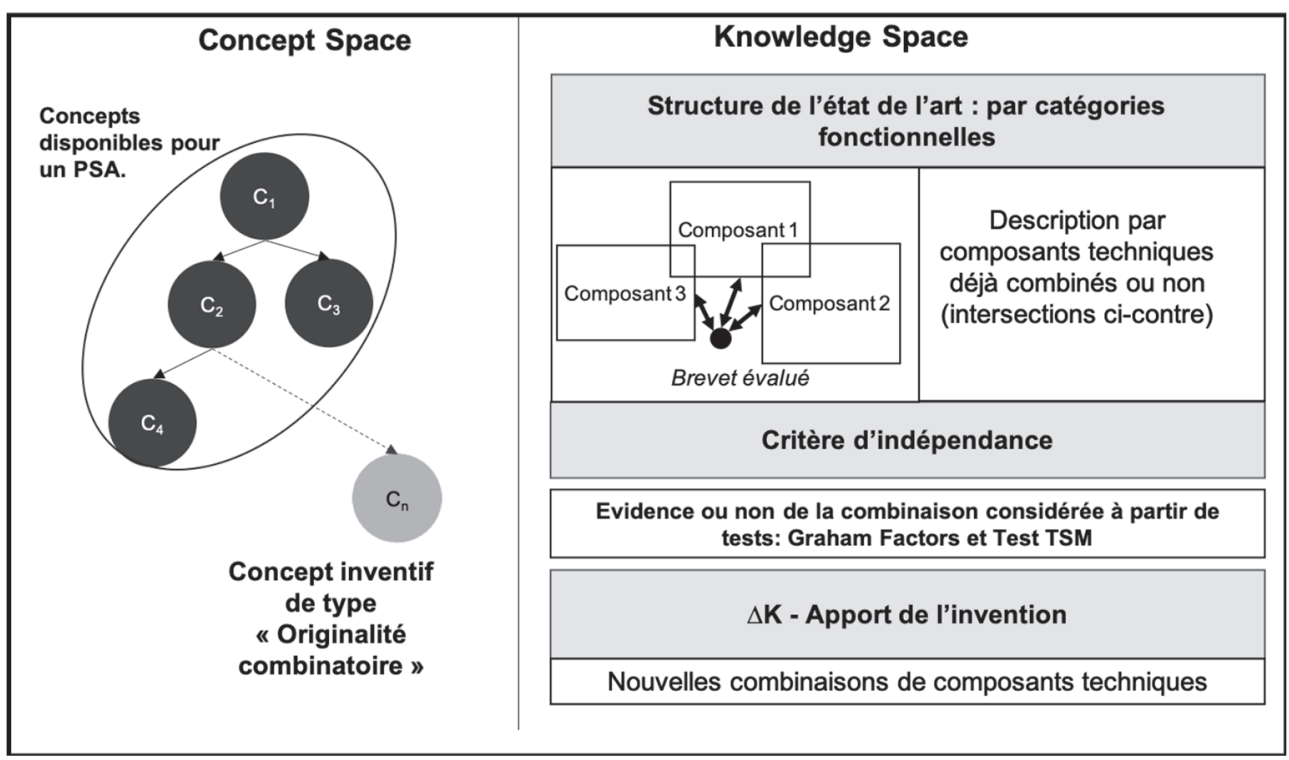

Figure 10. Synthèse du modèle d'invention pour le régime de l'originalité combinatoire (1941-2007) interprété d'après la théorie C/K

systèmes d'interrupteur prioritaire sont déjà connus, ainsi que le système de location de télévision. Le brevet apparaît donc comme un brevet de combinaisons. Cependant les critères d'évaluation de l'invention tels qu'ils apparaissent dans le régime du progrès fonctionnel ne sont pas utilisés. Concernant la question de l'évidence, les juges déclarent :

"Turning now to the determination of obviousness under section 103, we conclude that none of the references, either alone or in combination, would have disclosed or suggested to one of ordinary skill in the art the use of override switching means in a television rental system $»^{75}$.

Le test TSM prévoit donc que les juges ou les examinateurs de brevets doivent être capables d'identifier dans les références présentées des éléments suggérant à un homme du métier la combinaison revendiquée dans l'invention (" teachings of references can be combined only if there is some suggestion or incentive to do so »). Ce mode de raisonnement diffère radicalement des raisonnements antérieurs à 1952. L'invention n'est plus analysée en fonction de sa nature ou sa qualité (existence d'actions nouvelles, ou d'effets synergiques nouveaux), mais l'est en fonction de l'existence de suggestions dans l'état de l'art qui aurait mené à la combinaison.

Ce nouveau régime opte donc pour une représentation bien plus combinatoire des inventions : l'état de l'art est lui-même décrit au travers d'éléments combinés, éléments considérés comme des composants fonctionnels qu'il est alors possible d'agréger au sein des systèmes. L'apport de l'invention est lui-même formulé comme la combinaison de ces composants. La question de l'inventivité (et donc du critère d'indépendance) prend alors une forme nouvelle : il s'agit avant tout de déterminer s'il aurait été évident pour un homme du métier de combiner les composants pour l'obtention du système souhaité. Cette

\footnotetext{
${ }^{75}$ ACS Hospital Systems, Inc., Appellant/cross-Appellee v. Montefiore Hospital and Wells National Services Corporation, Appellees/cross-Appellants, 732 F.2d 1572 (Fed. Cir. 1984).
} 
évidence ou non-évidence est évaluée au travers de différents indices ou critères : les Graham Factors et, plus tard, le test TSM. Cette focalisation sur l'évidence ou nonévidence de la combinaison définit donc le développement jurisprudentiel de cette époque que nous appelons le régime de l'originalité combinatoire.

Ce régime s'étend jusqu'au début des années 2000. Or en 2007 la décision KSR v. Teleflex, abondamment commentée par les juristes américains ${ }^{76}$, semble encore une fois introduire des éléments de raisonnement nouveaux ; en particulier, le test TSM est largement remis en cause. Mais, plus encore, c'est le raisonnement purement combinatoire qui est nuancé, ce qui semble indiquer la possible émergence d'un nouveau régime.

La décision KSR v. Teleflex (2007) ${ }^{77}$ marque ainsi potentiellement une nouvelle rupture jurisprudentielle par rapport aux jugements précédents puisqu'elle étend largement les capacités de l'homme du métier : notre étude s'arrêtera donc à cette décision récente. Le jugement a trait à une invention, brevetée par KSR, pour une pédale d'accélérateur ajustable (en fonction de la position du conducteur) contrôlée par ordinateur au travers d'un système modulaire de capteurs. De 1986 à 2007, l'évaluation effectuée par les tribunaux fédéraux reposait essentiellement sur l'application du test TSM qui permettait d'établir l'étendue des combinaisons accessibles à l'homme du métier. En 2007, la Cour suprême réfute ce test, considéré comme une interprétation trop étroite du critère de non-évidence :
«Under the correct analysis, any need or problem known in the field and addressed by the patent can provide a reason for combining the elements in the manner claimed. Second, the appeals court erred in assuming that a person of ordinary skill in the art attempting to solve a problem will be led only to those prior art elements designed to solve the same problem. [...] It is common sense that familiar items may have obvious uses beyond their primary purposes, and a person of ordinary skill often will be able to fit the teachings of multiple patents together like pieces of a puzzle ${ }^{78}$.

La Cour élargit donc la capacité de l'homme du métier à tirer parti d'éléments de l'état de l'art en supposant que certains brevets, bien que résolvant un problème très différent de celui que cherche à résoudre l'homme du métier, peuvent tout de même être mobilisés dans leur recherche. Par ailleurs, la Cour valide la capacité de l'homme du métier à tester des combinaisons prédictibles s'il a une chance raisonnable de parvenir à la solution («obvious to try test ») :

"When there is a design need or market pressure to solve a problem and there are a finite number of identified, predictable solutions, a person of ordinary skill in the art has good reason to pursue the known options within his or her technical grasp $\gg{ }^{79}$.

Par conséquent, quand bien même il n'existe aucune combinaison similaire de pédale ajustable et de système de capteurs électroniques correspondants, plusieurs inventions très proches justifient l'évidence du brevet déposé par KSR par l'argument que la combinaison aurait été « évidente à tester»

\footnotetext{
${ }^{76}$ Voir C. A. Cotropia, «Predictability and Nonobviousness in Patent Law after KSR », Michigan Telecommunication and Technology Law Review, vol. 20, n² 2, 2013, p. 391-437 ; K. M. L. Hayes, « Three Years Post-KSR: A Practitioner's Guide to Winning Arguments on Obviousness and a Look at What May Lay Ahead », Northwestern Journal of Technology and Intellectual Property, vol. 9, n³, 2010, p. 243-262 ; G. S. Lunney Jr. et C. T. Johnson, « Not so Obvious After All: Patent Law's Nonobviousness Requirement, KSR, and the Fear of Hindsight Bias », Georgia Law Review, vol. 47, 2012, p. 41-111 ; N. A. Thomas, « Secondary Considerations in Nonobviousness Analysis: The Use of Objective Indicia Following KSR v. Teleflex », New York University Law Review, vol. 86, n 6, 2011, p. 2070-2111 ; J. P. Putney, "Are Secondary Considerations Still "Secondary"?: An Examination of Objective Indicia of Nonobviousness Five Years After KSR », Intellectual Property Brief, vol. 4, n² 2, 2012, p. 45-49.

${ }^{77}$ KSR Co. v. Teleflex, 550 U.S. 398, 127 S. Ct. 1727, 167 L. Ed. 2d 705, 2007 U.S. LEXIS 4745.

${ }^{78}$ Ibid.

${ }^{79}$ Ibid.
} 
par un homme du métier et donc prédictible. L'homme du métier est donc capable de concevoir de nouvelles combinaisons si les éléments de l'état de l'art sont suffisamment « proches » de la solution pour le justifier. Ces différents points suggèrent un raisonnement d'évaluation très différent, qui considère l'homme du métier comme doué de créativité ordinaire. En particulier, on ne se fie plus simplement à l'originalité (donc la fréquence) des combinaisons, mais plus exactement à leur probabilité. Si l'on conserve la notion de combinaison, elle s'enrichit d'un critère d'indépendance fondé sur la « prédictibilité » d'une solution technique, qui rompt avec l'approche jusqu'alors dominante. C'est pourquoi nous interrompons le régime de l'originalité combinatoire en 2007.

\section{DISCUSSION \\ ET PERSPECTIVES}

Cette étude précise la nature des transformations de la jurisprudence et leur impact sur les inventions enregistrées dans le système des brevets. Nous dégageons l'existence de trois régimes d'invention distincts correspondant à différents discours sur l'évaluation de l'invention. Nous souhaitons souligner deux résultats importants de cette analyse qui répondent aux questions de recherche de cet article.

Premièrement, il apparaît que l'évaluation au travers de la figure de l'homme du métier implique une manière très hétérogène de structurer l'état de l'art. Ainsi, l'évaluation passe d'une comparaison à une unique antériorité (1790-1851), à une comparaison par rapport à une catégorie fonctionnelle d'inventions (1851-1941) et enfin à un ensemble de composants considérés dépendants au travers des combinaisons existantes entre ceux-ci (1941-2007). Chacune de ces structures de l'état de l'art renvoie à différentes figures d'homme du métier mobilisées par les tribunaux, ce qui contribue à éclairer la nature des évolutions de cette figure et de ses capacités (capacité à ajouter des propriétés nouvelles à un objet dans le régime de la nouveauté, capacité à perfectionner des technologies d'une catégorie d'invention dans le régime du progrès fonctionnel, puis capacité à combiner des catégories d'invention déjà combinées dans le régime de l'originalité combinatoire) et de manière générale à comprendre les différentes ères jurisprudentielles déjà décrites par les auteurs américains ${ }^{80}$.

Cette observation est rendue possible par l'emploi de la théorie $\mathrm{C} / \mathrm{K}$ : on constate alors que les formes d'inconnu qu'explorent les inventeurs sont en fait rendues visibles par un ordonnancement particulier du connu ${ }^{81}$. D'un point de vue méthodologique, cela a des implications importantes sur les analyses historiques à partir des brevets : un brevet au début du $\mathrm{XIX}^{\mathrm{e}}$ siècle n'est a priori pas similaire à un brevet de la fin du $\mathrm{XX}^{\mathrm{e}}$ siècle puisqu'il recouvre des acceptions différentes de la notion d'invention. En particulier, toute analyse des brevets révèle nécessairement une double évolution : à la fois la dynamique de dépôts et la nature des inventions brevetées, mais également la nature des inventions sélectionnées et validées par les offices des brevets. Par ailleurs, l'influence de la structure de l'état de l'art sur les modèles d'invention apparaît majeure : ce n'est pas tant le raisonnement de l'inventeur lui-même qui détermine la forme de l'invention, mais bien plutôt le régime adopté pour la description des solutions techniques déjà connues. Cela

\footnotetext{
${ }^{80}$ R. K. Sawyer, « Creativity, Innovation, and Obviousness », art. cit.; J. F. Duffy, « Inventing Invention: a Case Study of Legal Innovation », art. cit.

${ }^{81}$ Du point de vue de la théorie $\mathrm{C} / \mathrm{K}$, on peut remarquer que la figure d'homme du métier joue un rôle singulier : elle permet aux évaluateurs de l'invention de contrôler simultanément l'opération de partition expansive (en permettant une analyse de l'expansion par rapport au connu qu'il met en scène) et l'opération de conjonction (en assurant un niveau de description suffisant pour considérer la solution obtenue comme une connaissance acquise et validée) : voir A. Hatchuel et B. Weil, « C-K Design Theory: an Advanced Formulation », art. cit.
} 
suppose que chaque modèle d'invention repose sur une organisation particulière du savoir technique, telle que formalisée dans les brevets. À ce titre, la classification des brevets, qui est le système d'organisation des brevets, devrait donc elle-même représenter ce qu'est l'invention. Les études les plus récentes de la classification soutiennent cette hypothèse : au début du XIX ${ }^{\mathrm{e}}$ siècle, c'est une classification par industrie qui est adoptée et renvoie essentiellement à des catégories d'objets utilisés dans chacune de ces industries; dès la fin du XIX ${ }^{\mathrm{e}}$ siècle, et la mise en place du département de classification (en 1898), apparaît une approche fonctionnelle, suivie d'une approche dite par "structure " (notamment utilisée en chimie) qui décrit les arrangements effectués ${ }^{82}$. Les théories de la classification répondent donc étonnamment aux théories de l'invention mobilisées par les juges, ce qui corrobore l'argument défendu dans notre analyse.

Deuxièmement, le régime de la nouveauté, le régime du progrès fonctionnel et le régime de l'originalité combinatoire reposent sur une historicisation de la notion d'invention: loin de renvoyer à un processus « inventif » unique qu'il s'agirait de décrire, on constate une accumulation progressive d'une variété de théories au sein du discours jurisprudentiel qui coïncident avec une rationalisation de l'activité d'évaluation de l'invention (comme le montrent les évolutions organisationnelles de l'office et de gestion de l'état de l'art). Cette rationalisation de l'évaluation stabilise des méthodes, des critères et des justifications de ce qui sera considéré comme « inventif» selon les périodes. Ce faisant, ces modèles d'invention définissent des types d'inconnus brevetables : l'invention n'est acceptée que parce qu'elle offre, dans une structure du connu donnée, une forme d'écart significatif, qui ne puisse être obtenu par des opérations déjà connues (renvoyant au critère d'indépendance). Cet ancrage historique de ce qu'est une invention éclaire sans doute l'approche essentiellement combinatoire adoptée par les auteurs récents ${ }^{83}$ et elle en montre la limite : s'il est sans doute possible de décrire de manière combinatoire toute solution technique, il est difficile de comprendre la nature « inventive » de certains brevets du $\mathrm{XIX}^{\mathrm{e}}$ siècle à partir de ce point de vue.

Ce rapprochement entre inconnu reconnu inventif et mise en ordre spécifique du connu met également en évidence une limite de notre étude : le découpage temporel de nos régimes d'invention provient de l'analyse généalogique effectuée à partir des discours des juges. Or ces discours portent sur des inventions, des objets distincts : il est donc probable que les discours observés portent également sur un état des connaissances techniques et scientifiques donné, dont la mobilisation correspond plus ou moins à une structure de connaissances donnée. Les régimes d'invention ne seraient donc pas organisés chronologiquement, mais plutôt associés à un état donné des connaissances en lien avec l'objet de l'invention. Partant de cette hypothèse, il n'y aurait pas une succession de ces régimes, mais plutôt une superposition; selon l'invention et l'état de l'art qui lui est associé, les juges adopteraient un certain raisonnement d'évaluation. À titre d'illustration, il est intéressant de constater que la biotechnologie pose aujourd'hui des problèmes importants d'évaluation, en particulier à cause de la particularité du savoir technique qui lui est associé : à cause de la forte variabilité des conditions de mise en œuvre des inventions biotechnologiques, l'approche combinatoire peine à fournir un cadre d'analyse propice à évaluer ce qui est atteignable par un homme du métier. C'est d'ailleurs la notion nouvelle de « prédictibilité » introduite dans la décision $K S R$ v. Teleflex qui a servi de guide

\footnotetext{
${ }^{82}$ L. Falasco, « Bases of the United States Patent Classification », World Patent Information, vol. 24, $\mathrm{n}^{\circ}$ 1, 2002 , p. 31-33; F. Lafond et D. Kim, « Long-run Dynamics of the US Patent Classification System », art. cit.

${ }^{83}$ W. B. Arthur, «The Structure of Invention », Research Policy, vol. 36, n 2, 2007, p. 274-287; H. Youn, D. Strumsky, L. M. A. Bettencourt, et J. Lobo, «Invention as a Combinatorial Process: Evidence from US patents », Journal of The Royal Society Interface, vol. 12, n 106, 2015.
} 
principal dans le cas controversé des brevets touchant la molécule Crispr ${ }^{84}$.

Les régimes d'invention et les modèles qui leur sont associés ne sont certes pas établis indépendamment des demandes de brevet de l'époque ; et il est donc fort possible qu'il existe une intime concordance entre les modèles d'invention mobilisés par les tribunaux et ceux que mobilisent les inventeurs. Cette histoire juridique de l'invention gagnerait donc à être éclairée et mise en lien avec une histoire plus approfondie des écosystèmes d'invention ou des systèmes de brevets au cours des $\mathrm{XIX}^{\mathrm{e}}$ et $\mathrm{XX}^{\mathrm{e}}$ siècles. En caractérisant chaque raisonnement d'évaluation de l'invention, il apparaît que les tactiques à développer pour « inventer » diffèrent probablement dans chaque régime d'invention. Bien que les résultats aient été établis à partir de données américaines, on peut donc plus généralement s'interroger sur les formes d'organisation de l'invention apparues en Amérique du Nord et en Europe (Etats-Unis, mais aussi France, Allemagne, Angleterre). Ceci permettrait de mettre en regard l'histoire du droit du brevet avec l'histoire de l'invention au sein des entreprises. On peut déjà noter quelques échos intéressants :

1) Le régime de la nouveauté implique une structure des connaissances par éléments ou par objets techniques. Or ceci apparaît relativement cohérent avec le développement du machinisme (filature mécanisée, machines à vapeur, chemins de fer) où l'invention technique semble être décrite comme l'émergence régulière de nouveaux objets. Ces inventions-objets font d'ailleurs le succès des inventeurs-entrepreneurs de l'Angleterre du début du $\mathrm{XIX}^{\mathrm{e}}$ siècle $^{85}$.

2) À partir de 1850 , on voit apparaître le régime du progrès fonctionnel : l'état de l'art est alors décrit en fonction de catégories d'objets, décrites par des fonctions techniques particulières. La capacité à obtenir une avancée fonctionnelle significative suppose donc des stratégies de recherche plus systématiques portant sur des catégories d'objets identifiées : on voit là apparaître les bureaux d'études en Allemagne (et les enseignements sur la conception des machines de Redtenbacher ${ }^{86}$ et, plus tard, les grands laboratoires industriels américains qui produisent des avancées fonctionnelles significatives sur des catégories « fonctionnelles » : télécommunications, réseaux électriques, électroniques (AT\&T, Bell Labs ${ }^{87}$.

3) Dès la seconde moitié du $X X^{\mathrm{e}}$ siècle, le régime de l'originalité combinatoire induit une représentation de l'état de l'art sous forme de composants fonctionnels, avec une vision de l'invention technique comme système organisé : cela correspond à l'apparition des clusters industriels, des grappes technologiques, des réseaux, qui précisément visent à̀ organiser des systèmes de connaissances facilitant les combinaisons originales, en s'appuyant sur une conception systématique ${ }^{88}$.

Pourrait-on voir ici une histoire croisée entre régimes d'invention (et droit du brevet)

\footnotetext{
${ }^{84}$ Voir J. S. Sherkow, «Inventive Steps: the CRISPR Patent Dispute and Scientific Progress », EMBO Reports, vol. $18, \mathrm{n}^{\circ} 7,2017$, p. 1047-1051.

${ }^{85} \mathrm{P}$. Le Masson et B. Weil, « La conception innovante », art. cit.

${ }^{86} I d$., «Aux sources de la R\&D : genèse des théories de la conception réglée en Allemagne (1840-1960) », Entreprises et Histoire, $\mathrm{n}^{\circ}$ 58, avril 2010, p. 11-50.

${ }^{87}$ L. S. Reich, The Making of American Industrial Research: Science and Business at GE and Bell, 1876-1926, Cambridge, Cambridge University Press, 2002.

${ }^{88}$ J.-B. Zimmermann, " Groupes industriels et grappes technologiques », Revue d'économie industrielle, vol. 47, $\mathrm{n}^{\circ} 1,1989$, p. 89-102 ; S. Yayavaram et G. Ahuja, « Decomposability in Knowledge Structures and its Impact on the Usefulness of Inventions and Knowledge-Base Malleability », Administrative Science Quarterly, vol. 53, $\mathrm{n}^{\circ} 2$, 2008, p. 333-362.
} 
et régimes de conception technique ? Dans tous les cas, ces échos forts entre les modèles formels de structures de connaissances liés à l'invention et les formes d'organisations industrielles au cours des $\mathrm{XIX}^{\mathrm{e}}$ et $\mathrm{XX}^{\mathrm{e}}$ siècles invitent à considérer l'impact important de modèles d'invention institutionnalisés au sein des systèmes de brevets sur le système industriel. De fait, on s'éloigne ici d'une histoire de l'invention technique qui traiterait des transformations des systèmes techniques (mettant l'accent sur des typologies d'objets dominants et les systèmes socio-économiques correspondants ${ }^{89}$ ) pour privilégier une histoire de l'invention relative à la transformation des raisonnements de conception (mettant plutôt l'accent sur les structures de connaissances et les efforts de conception rendus possibles au niveau de l'écosystème par cette structure de connaissances). D'un point de vue normatif, il est possible que la diversité des modèles présentés puisse être précisément un moyen de s'extraire de formes d'inconnus techniques dominants (correspondant à un régime relativement institutionnalisé) pour explorer de nouvelles voies techniques, qui n'étaient alors que peu visibles. D'un point de vue plus descriptif, on peut se demander si les études des techniques ne sont pas elles-mêmes le miroir de certaines représentations de l'invention : en effet toute description des techniques nécessite un effort d'ordonnancement ou de mise en relation des différents apports techniques qui puisse décrire l'évolution observée des techniques ; or cette évolution incorpore peut-être une représentation de ce qu'est l'effort d'invention technique, et donc de l'effort de conception de l'homme du métier. À titre d'exemple, en 1877, Franz Reuleaux introduit son œuvre Cinématique. Principes fondamentaux d'une théorie générale des machines par un état de l'art de la science des machines où se mêlent constamment des efforts de classification des machines et des principes généraux liés à leur génération ${ }^{90}$. Enfin, d'un point de vue plus prospectif, il est probable que la décision KSR v. Teleflex de 2007 révèle un nouveau déplacement de ce rapport entre inconnu et connu dans lequel se concrétise la notion d'invention : si un nouveau régime d'invention technique apparaît bien, ce sont alors sans doute un nouveau langage et de nouveaux outils de la conception technique qu'il faudrait également inventer.

\footnotetext{
${ }^{89}$ Voir B. Gille, « Prolégomènes à une histoire des techniques », Revue d'Histoire des Mines et de la Métallurgie, vol. 4, n 1-2, 1972, p. 3-65 ; L. Mumford, Technique et civilisation [1934], Paris, Le Seuil, 1950. Chez ces deux auteurs majeurs de l'histoire des techniques, les transformations observées portent plutôt sur la nature des systèmes techniques (émergence de nouvelles sources d'énergie ou de matières, de nouvelles techniques et expertises particulières et/ou d'appareils industriels qui leur sont associés).

${ }^{90}$ F. Reuleaux, Cinématique. Principes fondamentaux d'une théorie générale des machines, Paris, Librairie F. Savy, 1877, p. 11-20. L'auteur cite dans l'introduction de nombreux traités portant sur la science des machines en montrant qu'à partir de principes de construction des machines, de nombreux auteurs ont établi simultanément des systèmes de classification des machines.
} 